\title{
NOVEL NON-LINEAR ELASTIC STRUCTURAL ANALYSIS WITH GENERALISED TRANSVERSE ELEMENT LOADS USING A REFINED FINITE ELEMENT
}

\author{
C.K. $\mathrm{Iu}^{*, 1}$ and M.A. Bradford ${ }^{2}$ \\ ${ }^{1}$ School of Civil Engineering and Built Environment \\ Queensland University of Technology \\ QUT Brisbane, QLD, Australia \\ ${ }^{2}$ Centre for Infrastructure Engineering and Safety \\ School of Civil and Environmental Engineering \\ The University of New South Wales \\ UNSW Sydney, NSW, Australia \\ *(Corresponding author: E-mail: jerry.iu@qut.edu.au /iu.jerryu@gmail.com)
}

Received: 4 May 2014; Revised: 16 June 2014; Accepted: 8 July 2014

\begin{abstract}
In the finite element modelling of structural frames, external loads usually act along the elements rather than at the nodes only. Conventionally, when an element is subjected to these general transverse element loads, they are usually converted to nodal forces acting at the ends of the elements by either lumping or consistent load approaches. For a first- and second-order elastic analysis, the accurate displacement solutions of element load effect along an element can be simulated using neither lumping nor consistent load methods alone. It can be therefore regarded as a unique load method to account for the element load nonlinearly. In the second-order regime, the numerous prescribed stiffness matrices must indispensably be used for the plethora of specific transverse element loading patterns encountered. In order to circumvent this shortcoming, this paper shows that the principle of superposition can be applied to derive the generalized stiffness formulation for element load effect, so that the form of the stiffness matrix remains unchanged with respect to the specific loading patterns, but with only the magnitude of the loading (element load coefficients) being needed to be adjusted in the stiffness formulation, and subsequently the non-linear effect on element loadings can be commensurate by updating the magnitude of element load coefficients through the non-linear solution procedures. In principle, the element loading distribution is converted into a single loading magnitude at mid-span in order to provide the initial perturbation for triggering the member bowing effect due to its transverse element loads. This approach in turn sacrifices the effect of element loading distribution except at mid-span. Therefore, it can be foreseen that the load-deflection behaviour may not be as accurate as those at mid-span, but its discrepancy is still trivial as proved. This novelty allows for a very useful generalised stiffness formulation for a single higher-order element with arbitrary transverse loading patterns to be formulated. Moreover, another significance of this paper is placed on shifting the nodal solution (system analysis) to both nodal and element solution (sophisticated element formulation). For the conventional finite element method, such as cubic element, all accurate solutions can be only found at node. It means no accurate and reliable structural safety can be ensured within element, and as a result, it hinders the engineering applications.
\end{abstract}

Keywords: Elastic instability, Finite element, Transverse element load effect, Higher-order element formulation, Nodal solution, Element solution

\section{INTRODUCTION}

General load cases for framed structures, such as permanent loads, live loads and wind loads, usually involve patterns of loading which act transversely along the elements of the frame. It is usual in the finite element modelling to convert these loads to nodal loads, and to discretise the member into several elements, with the transverse loads taken account of as nodal forces in order to capture the first- and second-order structural response accurately in terms of nodal solution. However, when using one element for a member, it in turn means no accurate first-order (element load effect) and second-order (member bowing effect triggered by transverse element load) solutions is available except at the element nodes, as long as the assumed finite element function of an element excludes the condition of force equilibrium, such as cubic function. This paper is therefore concerned with the development of a numerical technique for incorporating transverse 
element loading in a sophisticated element formulation to replicate the accurate first- and second-order solutions along itself, when subjected to transverse element loads, and with the reducing of the difficulties encountered with the multiplicity of possible loading patterns and regimes to being represented by the stiffness formulation of a single element.

Kondoh et al. [1] presented a simplified procedure for the finite deformation analysis of space frames using one beam element to model each member, which involved the non-linear coupling of bending and stretching. Unfortunately, a few of elements were required for a single member in some reported examples for the accurate solutions by using the higher-order element approach. To this end, Chan and Zhou [2][3] developed a PEP finite element to simulate the second order effect on a member with an initial geometric imperfection. Izzuddin [4] subsequently formulated a fourth-order displacement-based finite element for structures under thermal loads, while Liew et al. [5] made use of a stability function formulation in their stiffness matrices so that geometric non-linearity in a member could be incorporated. Recently, Iu and Bradford [6][7][8] have developed the higher-order element using higher-order element, which showed the great applications of second-order inelastic framed structures.

Despite the advocacy of using a second-order analysis with a higher-order element approach, it seems a sophisticated element of this type which accounts for element loading has not been presented in the open literature, and either consistent or lumped load methods are used in lieu of incorporating transverse loading into the element formulation. The main drawback of using lumped and consistent loads is its inaccuracy, since it takes the form of a first- and second-order element loading response at node (nodal solution) by virtue of the system analysis; especially, the assumed finite element function does not satisfy the force condition. Because of this, most reported research has accounted for the coupling effect at the system level by merely dividing a member into a few elements to replicate the behaviour of a member by the accurate solutions at nodes of a few elements.

In order to account for the element load effect within a single element, Zhou and Chan [9][10] presented a second-order analysis that is capable of modelling the effects of element loads in the element stiffness formulation, in lieu of by a system analysis. However, each element loading pattern or regime requires a specific element stiffness matrix, which is limiting its applications because of the usual multiplicity of loading scenarios met in practice. To overcome this difficulty, a proficient and generalised element formulation is developed in this paper which facilitates the modelling of second-order loading effects covering a wide range of transverse loading regimes, which is founded on the principle of superposition of simple loading cases within a second-order analysis framework. The complex loading regimes are formed from these specific simple or fundamental loading cases, each of which is characterised by one representative bending moment coefficient. Consequently, the complex loading regime is defined in the stiffness coefficient by the combination of these moment coefficients only prior to present non-linear analysis. It means the magnitude of stiffness matrix representing the specific complex loading regime in lieu of stiffness matrix itself, which is updated with the recourse to the non-linear solution procedures for non-linear member bowing effect due to that complex element loading. Meanwhile, the principle of superposition is no longer effective in the course of non-linear solution procedures which is merely applied for deriving that non-linear stiffness formulation afore non-linear analysis. As such, the method is a trade-off between simplicity in the formulation and accuracy in describing the member buckling due to element load effect by virtue of the generalized element stiffness matrix. The ranges of the validity of the proposed non-linear analysis which incorporates element loading are illustrated through several examples chosen to illustrate its feasibility, versatility and accuracy. 


\section{ASSUMPTIONS}

The following assumptions are made in the formulation:

- The beam is prismatic and slender, with the Euler-Bernoulli hypothesis being valid;

- Warping deformations, shear deformations and the Wagner effect are neglected, so that lateral buckling is not considered;

- The loads increase and decrease incrementally and proportionally;

- The loading is conservative, with both nodal and element loading being admissible; and

- The strains are small but large displacements are included.

The transverse loading is not restricted as can occur in conventional finite element formulations, insofar as the lumped and consistent nodal approaches are not merely used to treat the transverse element load.

\section{DISPLACEMENT FUNCTION FOR HIGHER-ORDER BEAM-COLUMN ELEMENT}

The vector of deformations along an element are taken as $\mathbf{u}=\{u, v, w, \phi\}^{\mathrm{T}}$, which comprise the deformations $u$ in the longitudinal $x$-direction, $v$ in the $y$-direction, $w$ in the $z$-direction and the twist $\phi$ about the $x$-axis. Because the displacement functions for the element representation herein are referred to a co-rotational coordinate, the dependent variables for the transverse displacement $v$ and $w$ are replaced by the nodal rotations $\theta_{z}$ and $\theta_{y}$ about the $z$ and $y$-axes, respectively. These rotations are the dependent variables which define the transverse displacements in the element stiffness formulation which follows.

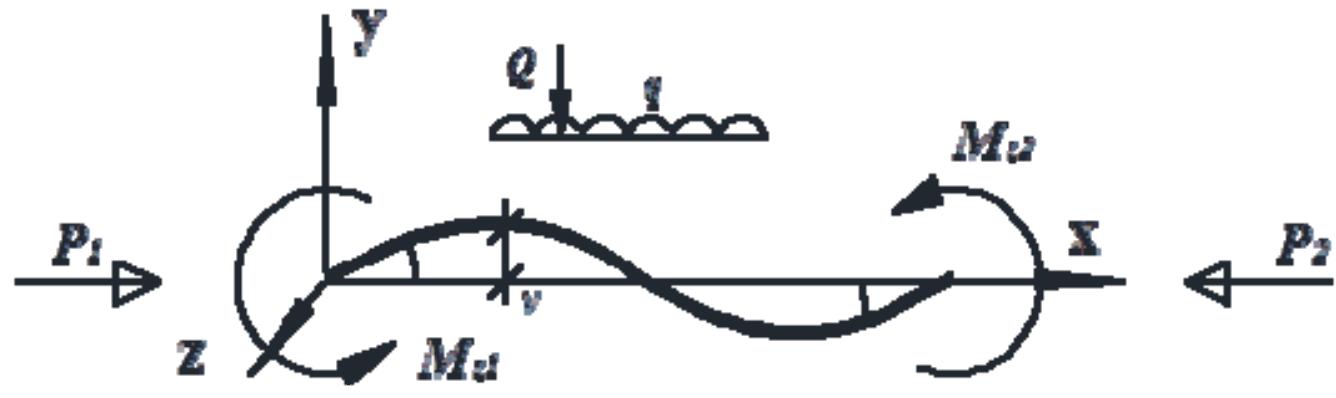

Figure 1. Equilibrium of Beam-column Element about z-axis under Element Loadings

External transverse element loads on an element generate additional non-linear effects that are manifested in the second-order element solution. To this end, the effect of transverse loading in the element is taken into account in the magnitude of element stiffness formulation, in which a relationship between the deflections and the loading under transverse element loads is modelled accurately and adequately using a single element. As a result, apart from satisfying the primary kinematic boundary conditions, the displacement function proposed which includes the general transverse element distributed loading $q$ and concentrated loading $Q$ shown in Figure 1 can be derived by satisfying the secondary statical boundary of force equilibrium. Without loss of generality, the mid-span moment $M_{0}$ obtained by superimposing the loading effects using elementary force statics, is used in the equilibrium condition for moments about the $z$ - and $y$-axes; this superposition being valid prior to the commencement of the non-linear analysis. Further, the second-order moments $P v$ and $P w$ due to the member $\mathrm{P}-\delta$ effects are also introduced into the equilibrium equation when equilibrium is formulated along the element instead of at the end nodes of the element. It is therefore helpful to incorporate the member bowing and element load effects 
into the element stiffness formulation based on a single element, whose higher-order elastic displacement function is derived in the following.

Linear functions are assumed for the axial deformation and twist; pure axial deformation and twist are assumed as being independent of the element load effect, so that

$u=(1-\xi) u_{1}+\xi u_{2} \quad$ and $\quad \phi=(1-\xi) \theta_{x 1}+\xi \theta_{x 2}$,

in which $u=u_{1}$ at $x=0, u=u_{2}$ at $x=L$ are the axial nodal deformations, $\phi=\theta_{x 1}$ at $x=0, \phi=\theta_{x 2}$ at $x=L$ are the twist nodal deformations, and where $\xi=x / L$. In order to include the member bowing effect and transverse element loading in a single element, the kinematic boundary conditions about the $y$-direction are

$v=\left\{\begin{array}{ll}0 & x=0 \\ 0 & x=L\end{array} \quad\right.$ and $\quad \frac{\partial v}{\partial x}= \begin{cases}\theta_{z 1} & x=0 \\ \theta_{z 2} & x=L,\end{cases}$

while the equation of bending given by

$E I_{z} \frac{\partial^{2} v}{\partial x^{2}}=P v-M_{z 1}(1-\xi)+M_{z 2} \xi+M_{0}$

which produces

$E I_{z} \frac{\partial^{2} v}{\partial x^{2}}=P v+\frac{M_{z 2}-M_{z 1}}{2}+M_{0} \quad$ at $\quad \xi=1 / 2$,

leads to the deflection

$$
\begin{aligned}
v= & {\left[\xi-\frac{4(24+\omega) \xi^{2}}{48+\omega}+\frac{(48+5 \omega) \xi^{3}}{48+\omega}-\frac{2 \omega \xi^{4}}{48+\omega}\right] L \theta_{z 1}+\left[\frac{-(48-\omega) \xi^{2}}{48+\omega}+\frac{3(16-\omega) \xi^{3}}{48+\omega}+\frac{2 \omega \xi^{4}}{48+\omega}\right] L \theta_{z 2} } \\
& -\frac{\bar{M}_{0} L}{(48+\omega)}\left[\xi^{2}-2 \xi^{3}+\xi^{4}\right]
\end{aligned}
$$

or

$v=N_{1} L \theta_{z 1}+N_{2} L \theta_{z 2}+N_{q} L \bar{M}_{0}$,

in which

$\omega=\frac{P L^{2}}{E I}$

is a dimensionless axial load parameter and $N_{1}, N_{2}$ and $N_{q}$ are displacement functions with respect to the first and second node rotations, and element loads, respectively. The equivalent mid-span moment $\bar{M}_{0}$ for a variety of element loads is given in Appendix 1, which represents the amount of the equivalent mid-span moment produced by various element loads and derived analytically from a force equilibrium equation. The transverse displacement $v$ in the $z$ direction can be similarly defined. 
An elementary verification of the functions in Eqs. 5 and 6 can be established for a fixed beam under a point load applied at mid-span, for which $\theta_{1}$ and $\theta_{2}$ are zero and using $a=L / 2$ by Eqs. 72 or 73 in Appendix 1 reduces to the exact theoretical solution for the mid-span deflection of $Q L^{3} / 192 E I$ $(x=L / 2)$. Similarly for a uniformly distributed load instead of a point load at mid-span, the displacement function produces the exact mid-span deflection of $q L^{4} / 384 E I$ using Eq. 76 in Appendix 1. Further theoretical verifications of displacement function for more general load distributions are discussed in Section 6.

It should be noted that the higher-order displacement function $N_{q}$ in Eq. 5 is independent of the loading regime along the element. The different element load solutions for different loading regimes is merely incorporated into the equivalent moment $\bar{M}_{0}$ with respect to mid-span given in Appendix 1 which does not depend on the independent variable $x$, but on the magnitude of the loading and the point of application of the load with respect to the mid-span location. This significantly implies that the different element loading regimes vary with the magnitudes of stiffness matrix in lieu of stiffness matrix itself, and so only the fundamental load cases listed in Appendix 1 are needed to customize complex loading regimes in the second-order analysis. The distribution of complex loading regimes is therefore condensed into the magnitude of stiffness matrix in terms of equivalent moment $\bar{M}_{0}$ at mid-span, for which provides the initial perturbation for triggering the member bowing effect due to its transverse element loads.

\section{STIFFNESS FORMULATION FOR HIGHER-ORDER BEAM-COLUMN ELEMENT}

The internal strain energy $U$ caused by the axial strain $\varepsilon_{x}$ and twist strain $\gamma_{x}$ in the element continuum is considered in order to formulate the stiffness matrices in the present second order elastic beam-column element. It is routinely given by

$U=\frac{1}{2} \int_{V o l}\left(E \varepsilon_{x}^{2}+G \gamma_{x}^{2}\right) d(V o l)$

which can be expressed as [6]

$$
\begin{aligned}
U & =\frac{1}{2} E A \int_{L}\left(\frac{\mathrm{d} u}{\mathrm{~d} x}\right)^{2} \mathrm{~d} x+\frac{1}{2} P \int_{L}\left(\frac{\mathrm{d} v}{\mathrm{~d} x}\right)^{2} \mathrm{~d} x+\frac{1}{2} P \int_{L}\left(\frac{\mathrm{d} w}{\mathrm{~d} x}\right)^{2} \mathrm{~d} x \\
& +\frac{1}{2} E I_{z} \int_{L}\left(\frac{\mathrm{d}^{2} v}{\mathrm{~d} x^{2}}\right)^{2} \mathrm{~d} x+\frac{1}{2} E I_{y} \int_{L}\left(\frac{\mathrm{d}^{2} w}{\mathrm{~d} x^{2}}\right)^{2} \mathrm{~d} x+\frac{1}{2} G J \int_{L}\left(\frac{\mathrm{d} \phi}{\mathrm{d} x}\right)^{2} \mathrm{~d} x
\end{aligned}
$$

in which $E A$ is the axial rigidity, $E I_{y}$ and $E I_{z}$ the flexural rigidities about the $y$ and $z$-axes respectively, $G J$ the torsional rigidity, $P$ the axial force; and $E$ is the elastic modulus and $G$ the shear modulus.

In this study, external loads are produced by nodal force vectors $\mathbf{f}_{k}$ and element load vectors $\boldsymbol{\Phi}_{k}$, so that the external work done $V$ comprises of two components. The first of these is the work done by the nodal forces $\mathbf{f}_{k}$ in moving through nodal displacements $\mathbf{u}_{k}$, while the second is the work done by the transverse element load $\Phi_{k}$ moving through the assumed transverse displacement field associated with the element displacement function vector $\mathbf{N}$ over the element length, in which $\mathbf{u}_{k}=$ $<u, \theta_{z 1}, \theta_{z 2}, \theta_{x}, \theta_{y 1}, \theta_{y 2}>^{\mathrm{T}}$ with $u=u_{1}-u_{2}$ and $\theta_{x}=\phi_{1}-\phi_{2}$. The principle of superposition can be 
applied to simplify the effect of the element load $\Phi_{k}$ on the external work $V$, for which in accordance with the assumption of conservative loading the work done $V$ caused by the element load vector $\Phi_{k}$ moving through the element deflections represented by $\mathbf{N}$ is independent of the axial load $P$ (and thus axial load parameter $\omega$ ) throughout; hence setting $\omega=0$ gives

$$
V=\int_{L} \mathbf{u}_{k}^{\mathrm{T}} \mathbf{N}^{\mathrm{T}} \boldsymbol{\Phi}_{k} \mathbf{d} x+\mathbf{u}_{k}^{\mathrm{T}} \mathbf{f}_{k}
$$

The elastic force-displacement relationship is derived from the total potential energy $\Pi$ of the general beam-column element subjected to both nodal and element loads. For second-order analysis, the total potential is the sum of the internal strain energy in Eq. 9 and external work done in Eq. 10, giving

$$
\begin{aligned}
\Pi= & \frac{E A}{2} \int_{L}\left(\frac{\mathrm{d} u}{\mathrm{~d} x}\right)^{2} \mathrm{~d} x+\frac{P}{2} \int_{L}\left(\frac{\mathrm{d} v}{\mathrm{~d} x}\right)^{2} \mathrm{~d} x+\frac{P}{2} \int_{L}\left(\frac{\mathrm{d} w}{\mathrm{~d} x}\right)^{2} \mathrm{~d} x+\frac{E I_{z}}{2} \int_{L}\left(\frac{\mathrm{d}^{2} v}{\mathrm{~d} x^{2}}\right)^{2} \mathrm{~d} x \\
& +\frac{E I_{y}}{2} \int_{L}\left(\frac{\mathrm{d}^{2} w}{\mathrm{~d} x^{2}}\right)^{2} \mathrm{~d} x+\frac{G J}{2} \int_{L}\left(\frac{\mathrm{d} \phi}{\mathrm{d} x}\right)^{2} \mathrm{~d} x-\int_{L} \mathbf{u}_{k}^{\mathrm{T}} \mathbf{N}^{\mathrm{T}} \mathbf{\Phi}_{k} \mathbf{d} x-\mathbf{u}_{k}^{\mathrm{T}} \mathbf{f}_{k} .
\end{aligned}
$$

The strain energy functional in Eq. 9 depends not only on the variables $\mathbf{u}_{k}$ but also on the axial load parameter $\omega$. Hence from Castigliano's first theorem of strain energy, the secant stiffness matrix is obtained from

$$
\mathbf{K}_{s}=\frac{\partial U}{\partial \mathbf{u}_{k}}+\frac{\partial U}{\partial \omega} \cdot \frac{\partial \omega}{\partial \mathbf{u}_{k}}
$$

This then leads to

$$
M_{\alpha 1}=\frac{\partial U}{\partial \theta_{\alpha 1}}=\frac{E I_{\alpha}}{L}\left(C_{1} \theta_{\alpha 1}+C_{2} \theta_{\alpha 2}+C_{q} \bar{M}_{0}\right)
$$

in which

$$
\begin{aligned}
& C_{1}=\frac{9216+3456 \omega / 5+68 \omega^{2} / 5+8 \omega^{3} / 105}{(48+\omega)^{2}} \\
& C_{2}=\frac{4608+576 \omega / 5+2 \omega^{2}+\omega^{3} / 42}{(48+\omega)^{2}} \\
& C_{q}=\frac{\omega^{2}}{210(48+\omega)^{2}}
\end{aligned}
$$

and to

$M_{\alpha 2}=\frac{\partial U}{\partial \theta_{\alpha 2}}=\frac{E I_{\alpha}}{L}\left(C_{1} \theta_{\alpha 1}+C_{2} \theta_{\alpha 2}-C_{q} \bar{M}_{0}\right)$, 
in which $(\cdot)_{\alpha}=(\cdot)_{y}$ or $(\cdot)_{z}$ as appropriate. Eq. 12 also leads to

$$
\begin{aligned}
P & =P_{1}-P_{2}=\frac{\partial U}{\partial e}+\frac{\partial U}{\partial \omega} \cdot \frac{\partial \omega}{\partial e} \\
& =E A\left[\frac{e}{L}+\sum_{\alpha=y, z}\left(C_{b}+b_{q 1}\left(\theta_{\alpha 1}-\theta_{\alpha 2}\right) \bar{M}_{0}+b_{q 2} \bar{M}_{0}^{2}\right)\right]
\end{aligned}
$$

in which $e=u=u_{1}-u_{2}, P_{i}$ is the axial load at $i$-th node and

$$
\begin{aligned}
& C_{b}=b_{1}\left(\theta_{\alpha 1}+\theta_{\alpha 2}\right)^{2}+b_{2}\left(\theta_{\alpha 1}-\theta_{\alpha 2}\right)^{2} \\
& b_{1}=\frac{6(48)^{2} / 5+18(48) \omega / 5+126 \omega^{2} / 35+\omega^{3} / 40}{(48+\omega)^{3}} \\
& b_{2}=\frac{2(48)^{2}+14(48) \omega / 5+66 \omega^{2} / 35+11 \omega^{3} / 840}{(48+\omega)^{3}} \\
& b_{q 1}=\frac{16 \omega}{35(48+\omega)^{3}} \\
& b_{q 2}=-\frac{12 / 35+\omega / 105}{(48+\omega)^{3}} .
\end{aligned}
$$

It can be seen the internal strain energy $U$ is load-dependent, so that coupling of the external element load and the element deformations is inherent in the present non-linear stiffness formulation of Eqs. 13 to 18.

Again, it is noteworthy that despite there being a vast range of possible element loading pattern, the line integration with respect to $x$ in Eq. 11 is essentially unchanged against a plethora of transverse element loads, because the use of principle of superposition prior to non-linear procedures separates the element load effect from deformations along an element $N_{q}$. As a result, the element load effect merely depends on the magnitude of the term $\bar{M}_{0}$ associated with the particular loading pattern, as given in Appendix 1. This salient feature provides a crucial insight into the generalized stiffness matrix of an element for a member regardless of a diverse element load cases instead of the magnitude of the term $\bar{M}_{0}$ being formulated in the non-linear stiffness formulation. The nonlinearity of element load effect can be traced through the magnitude of element load $\bar{M}_{0}$ through incremental load factor $\Delta \lambda_{i}$ in the nonlinear solution procedures. This feature avoids the need for tedious and numerous stiffness matrices under a plethora of general element loading patterns, leading to a simple, versatile and generalized stiffness formulation. The secant stiffness coefficients $C_{q}, b_{q 1}$ and $b_{q 2}$ which account for the element loading therefore vary between different loading patterns by altering the magnitude $\bar{M}_{0}$ only.

The coefficient $C_{q}$ induces the second-order moment due to the coupling of both the lateral element loads and the axial loads, whereas $b_{q 1}$ and $b_{q 2}$ quantify the axial force effect from this coupling. However, when there is no axial force and so $\omega=0, b_{q 1}$ and $b_{q 2}$ are 0 and $-12 / 3,870,720 \approx-3.1 \times 10^{-6}$ respectively. The last term $b_{q 2} \bar{M}_{0}^{2}$ may still be of certain contribution to axial resistance $P$ due to 
element load that in turn represents elongation caused by element load because of the squaring of $\bar{M}_{0}$. It should be emphasized that in most previous research on non-linear analysis, the coupling effect between the lateral load and the element stiffness has been neglected in non-linear finite element formulations.

The large deformations and the inclusion of the axial force parameter $\omega$ into the element formulation herald a potential situation for which convergence may be somewhat difficult. In addition, the member axial force term $\omega$ involves the bowing functions $b_{1}$ and $b_{2}$, which in turn are functions of $\omega$. Hence, Eq. 18 can be written in the form

$\Omega(\omega)=\frac{\omega I}{A L^{2}}-\frac{e}{L}-\sum_{\alpha=y, z}\left[b_{1}\left(\theta_{\alpha 1}+\theta_{\alpha 2}\right)^{2}+b_{2}\left(\theta_{\alpha 1}-\theta_{\alpha 2}\right)^{2}+b_{q 1}\left(\theta_{\alpha 1}-\theta_{\alpha 2}\right) \bar{M}_{0}+b_{q 2} \bar{M}_{0}^{2}\right]$

in which $\omega$ is the only unknown. The iterative procedure for which an equilibrium condition is sought, as also mentioned by Chan and Zhou [3] and Kassimali [11], proceeds by letting $\omega_{i}$ be an approximate solution of this equation $\Omega(\omega)$, which can measure the equilibrium condition within the element formulation. The first order Taylor expansion of this equation $\Omega(\omega)$ is

$\Omega\left(\omega_{i}+\Delta \omega_{i}\right)=\Omega\left(\omega_{i}\right)+\Omega^{\prime}\left(\omega_{i}\right) \Delta \omega_{i}$,

in which $(\cdot)^{\prime}=\mathrm{d}(\cdot) / \mathrm{d} \omega . \quad$ Further, from Eq. 24

$\Omega^{\prime}(\omega)=H=\frac{I}{A L^{2}}-\sum_{\alpha=y, z}\left[b_{1}^{\prime}\left(\theta_{\alpha 1}+\theta_{\alpha 2}\right)^{2}+b_{2}^{\prime}\left(\theta_{\alpha 1}-\theta_{\alpha 2}\right)^{2}+b_{q 1}^{\prime}\left(\theta_{\alpha 1}-\theta_{\alpha 2}\right) \bar{M}_{0}+b_{b 2}^{\prime} \bar{M}_{0}^{2}\right]$

in which the expression for $H$ also forms a part of the stiffness coefficients in the tangent stiffness matrix given subsequently, and also $H, b_{q 1}^{\prime}$ and $b_{q 2}^{\prime}$ are also given in Appendix 2 . It is interesting to note that the bowing function $b_{1}$ is stationary with respect to $\omega$. An updated value of $\omega$ is thus obtained from

$\omega_{i+1}=\omega_{i}+\Delta \omega_{i}=\omega_{i}-\frac{\Omega\left(\omega_{i}\right)}{H}$.

The tangent stiffness matrix is obtained by taking the second derivative of the total potential functional in Eq. 11 with respect to the variables $\mathbf{u}_{k}$ and axial load parameter $\omega$. When the work done $V$ is linear, this differentiation results in

$\mathbf{K}_{t}=\frac{\partial^{2} \Pi}{\partial \mathbf{u}_{j} \partial \mathbf{u}_{k}}=\frac{\partial}{\partial \mathbf{u}_{j}}\left(\frac{\partial U}{\partial \mathbf{u}_{k}}\right)+\frac{\partial}{\partial \mathbf{u}_{k}}\left(\frac{\partial U}{\partial \omega} \cdot \frac{\partial \omega}{\mathbf{u}_{j}}\right)$.

The tangent stiffness matrix of the beam-column element incorporating the response of the element load derived in this way is 


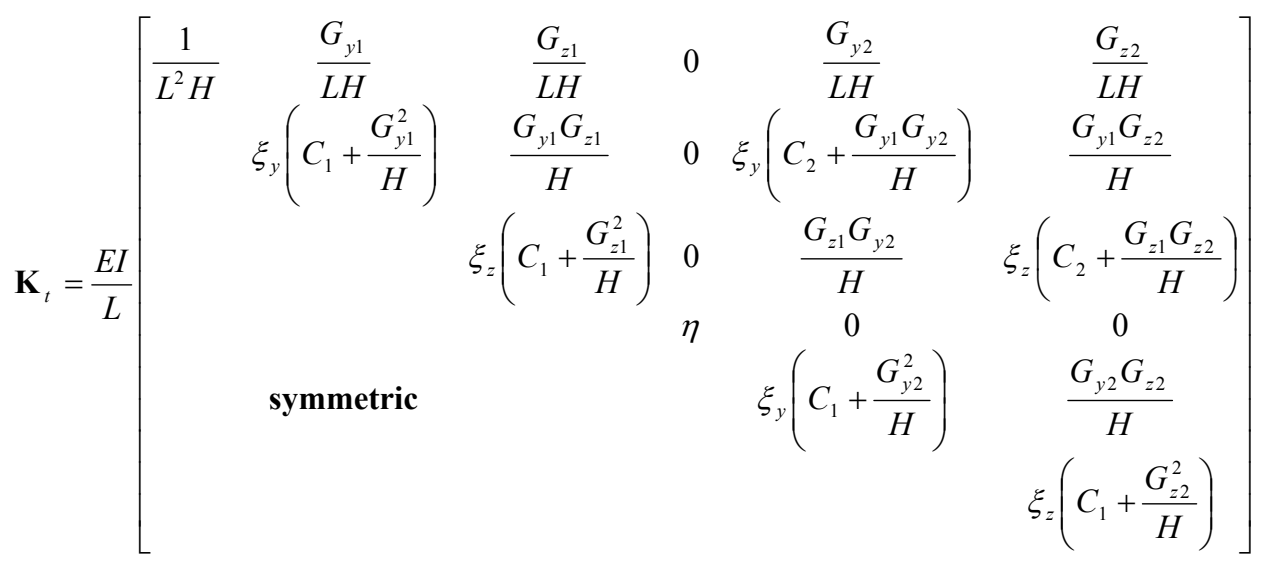

in which $\eta$ is torsional rigidity of $\left(G J+P r^{2}\right) / E I$ and it relates the incremental deformation to the corresponding external loads applied to an element in the member coordinates, in which $K_{i j}, G_{\alpha i}$ and $H$ are given in Appendix 2, $I$ is the relevant second moment of area about which bowing is considered and $\xi_{\alpha}=I_{\alpha} / I(\alpha=y$ or $z)$.

The tangent stiffness matrix needed for assembly and transformations in global coordinates $\mathbf{K}_{T}$ is

$$
\mathbf{K}_{T}=\sum_{\text {elements }} \mathbf{L} \mathbf{K}_{e} \mathbf{L}^{\mathbf{T}}=\sum_{\text {elements }} \mathbf{L}\left(\mathbf{T}^{\mathrm{T}} \mathbf{K}_{t} \mathbf{T}+\mathbf{M}_{\sigma}\right) \mathbf{L}^{\mathrm{T}}
$$

in which $\mathbf{T}$ is the transformation matrix relating the member forces to the element forces in local coordinates, $\mathbf{L}$ is the transformation matrix from the local coordinates to the global coordinates and $\mathbf{M}_{\sigma}$ is a stability matrix to allow for the work done by rigid body motions or the change of geometry of the structures as also shown in [12]. Because of the nature of the non-linearity in Eq. 11 , an incremental-iterative solution procedure is needed to trace the non-linear equilibrium path, including the non-linearity due to transverse element load effect.

\section{ILLUSTRATION OF ELEMENT LOAD EFFECT}

Figure 2 illustrates the theoretical principle of load lumping numerical procedures using the conventional finite element. A transverse element point load $\Delta Q$ is firstly applied at mid-span at a node between two elements, as in Figure 2(a). The deflection of the beam is such that its load-deflection response satisfies the tangent stiffness relationship; there is no axial deformation at the support as indicated in Figure 2(b) because there is no axial component initially in the tangent stiffness in the context of the conventional finite element method. In Figure 2(c), the secant stiffness determines the member resistance in accordance with the deformations of the finite elements (transverse deflections only); the axial force $P$ results from the extension of the element due to deflection alone which attempts to balance the external point load $\Delta Q$ by its vertical component due to the slightly deflected geometry; and thereby the unbalanced axial force appears in the next iteration. In the second iteration in Figure 2(d), the axial deformation $e$ (longitudinal movement) at the roller end is computed from the tangent stiffness relationship corresponding to the unbalanced axial force $P$ component. In Figure 2(e), the unbalanced axial force from the first iteration caused by the axial member force $P$ is cancelled by axial resistance from the secant stiffness relationship in accordance with the axial deformation $e$; equilibrium is achieved only if the convergence criterion is satisfied. In summary, the conventional finite element using lumping load method requires at least two elements and iterations to achieve equilibrium for this simple 
beam so as to include the element load response. Equilibrium can only be achieved through global system analysis, and so the element load response is solved at the global level using the conventional finite element method.

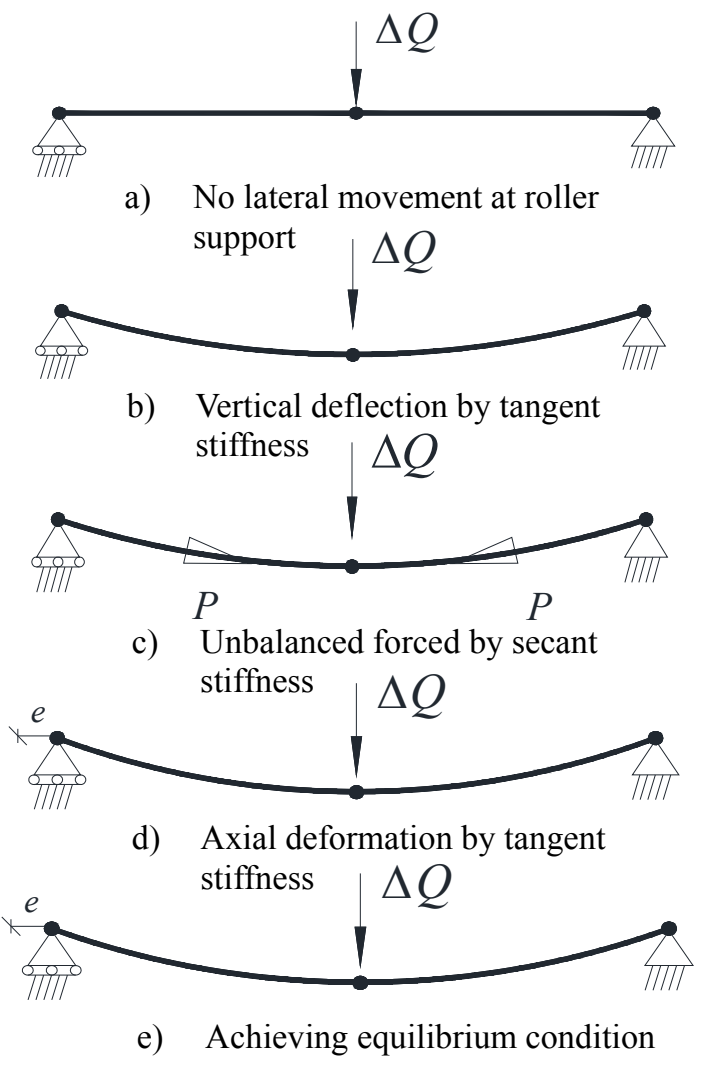

Figure 2. Numerical Procedures using the Conventional Finite Element Method

According to the present element load approach, once the transverse element point load $\Delta Q$ is applied at the mid-span of the single element used to model a simply-supported beam (Figure 3(a)), the axial deformation $e$ is computed from the tangent stiffness equation (Figure 3(b)). Despite there being no axial external load or unbalanced force component at the first iteration, the terms involving the coupling between the rotations $\theta$ and the axial deformation $e$ in the tangent stiffness matrix $\mathbf{K}_{T}$ in Eq. 29 allow for the axial deformations of the element to be computed according to vertical component of point load $\Delta Q$. Subsequently, the axial member force $P$ (Figure 3(c)) in Eq. 18 is self-equilibrated which is determined from the secant stiffness formulation $\mathbf{K} s$ in Eqs. 13 to 18 and which encompasses the axial effect through $e / L$, the flexural effect through $\theta$ as well as the element load effect through $\bar{M}_{0}$ and thereby maintains equilibrium at the element level; hence no unbalanced force is induced for the next iteration. Therefore, one iteration is theoretically adequate to achieve equilibrium for this simple beam subjected to element load, and it leads to efficient numerical convergence.

For simply speaking, the conventional finite element method accounting for the element load effect is reliant of the system analysis, whereas the present approach for the element load effect resorts to the sophisticated element stiffness formulation within element level, into which the element load in terms of $\bar{M}_{0}$ is incorporated. 


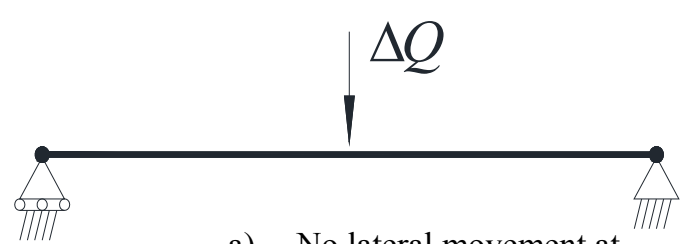

a) No lateral movement at roller support

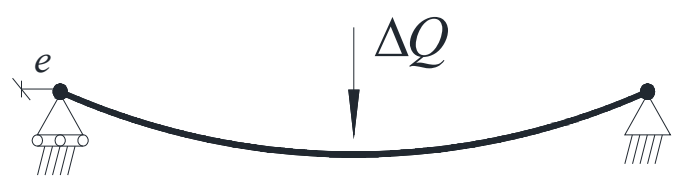

b) Deformations by tangent stiffness

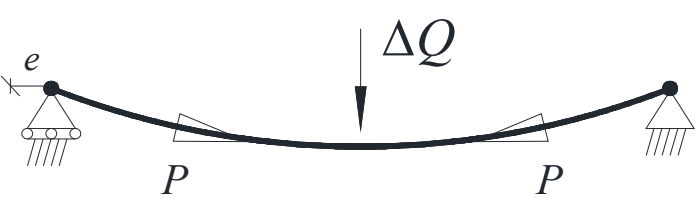

c) Achieving equilibrium condition

Figure 3. Numerical Procedures using the Present Approach

\section{NUMERICAL VERIFICATIONS}

This section firstly validates the displacement function for an element, for which the deflections obtained with the first-order effects of transverse load are compared with exact analytical results from the linear elastic method. A simple beam subjected to various regimes of transverse load using second-order analysis with or without axial load is then investigated. Following this, two small-scale elastic framed structures are investigated using the second-order procedure; one is a right-angled frame and the other a two-storey frame under uniform loading in which P- $\Delta$ effects take place. In these validation studies, a single element is used for each member of the framed structures in order to study the element solution.

\subsection{Deflections of a Prismatic Beam}

\subsubsection{Propped cantilever subjected to a point load}

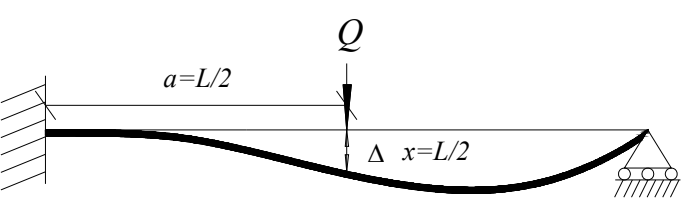

Figure 4. A propped Cantilever subjected to a Mid-span Point Load

Figure 4 shows a propped cantilever subjected to a concentrated load $Q$ at mid-span, for which the theoretical mid-span deflection is $\Delta=-(7 / 768)\left(Q L^{3} / E I\right)$, of which is derived from the linear elastic analytical method (e.g. unit load method). 
Using the consistent load method with a cubic element, the consistent load with respect to a released freedom, as well as the corresponding rotation, is

$\frac{4 E I \theta_{2}}{L}=\frac{Q L}{8} ; \quad \theta_{2}=\frac{Q L^{2}}{32 E I}$,

and using

$v=\left(1-\frac{3 x^{2}}{L^{2}}+\frac{2 x^{3}}{L^{3}}\right) v_{1}+\left(x-\frac{2 x^{2}}{L}+\frac{x^{3}}{L^{2}}\right) \theta_{1}+\left(\frac{3 x^{2}}{L^{2}}-\frac{2 x^{3}}{L^{3}}\right) v_{2}+\left(-\frac{x^{2}}{L}+\frac{x^{3}}{L^{2}}\right) \theta_{2}$

at $x=L / 2$ produces

$\left.v\right|_{x=L / 2}=\frac{Q L^{2}}{32 E I}\left(-\frac{L}{8}\right)=-\frac{Q L^{3}}{256 E I}$,

which is $57 \%$ different from the exact result. Using the higher-order element of this paper with the element load, when the axial force parameter $\omega=0$, the functions $N_{1}$ and $N_{2}$ in Eqs. 5 and 6 are the same as those of a cubic element. The function $N_{q}$ can calibrate its element solution due to element load from cubic element, and using Eqs. 52 or 53 in $N_{q}$ (Appendix 1). Eq. 5 produces

$$
\left.v\right|_{x=L / 2}=-\frac{Q L^{3}}{256 E I}-\frac{\left(4 Q L^{2} / E I\right) L}{48}\left[\left(\frac{1}{2}\right)^{2}-2\left(\frac{1}{2}\right)^{3}+\left(\frac{1}{2}\right)^{4}\right]=-\frac{7 Q L^{3}}{768 E I}
$$

which is the same as the exact result. It can therefore be seen that the higher-order element load component $N_{q}$ produces the exact solution, but using a cubic interpolation polynomial yield an answer that differs $57 \%$ from the exact one.

\subsubsection{Simply supported beam subject to a point load}

Figure 5 shows a beam subjected to a concentrated load at either a third point or at mid-span. For a load at mid-span (Figure 5(a)), the theoretical deflection is $\Delta=-(1 / 48)\left(Q L^{3} / E I\right)$. The consistent load and nodal end rotations are obtained from

$$
\frac{E I}{L}\left[\begin{array}{ll}
4 & 2 \\
2 & 4
\end{array}\right]\left\{\begin{array}{l}
\theta_{1} \\
\theta_{2}
\end{array}\right\}=\left\{\begin{array}{c}
-Q L / 8 \\
Q L / 8
\end{array}\right\} ; \quad\left\{\begin{array}{l}
\theta_{1} \\
\theta_{2}
\end{array}\right\}=\left\{\begin{array}{c}
-3 Q L^{2} / 48 E I \\
3 Q L^{2} / 48 E I
\end{array}\right\}
$$

and using a cubic element, Eq. 32 produces

$$
\left.v\right|_{x=L / 2}=-\frac{3 Q L^{2}}{48 E I}\left(\frac{L}{8}\right)+\frac{3 Q L^{2}}{48 E I}\left(-\frac{L}{8}\right)=-\frac{Q L^{3}}{64 E I},
$$

which is $25 \%$ different from the correct result. However, using Eqs. 52 or 53 in $N_{q}$ (Appendix 1). Eq. 5 produces

$$
\left.v\right|_{x=L / 2}=-\frac{Q L^{3}}{64 E I}-\frac{\left(4 Q L^{2} / E I\right) L}{48}\left[\left(\frac{1}{2}\right)^{2}-2\left(\frac{1}{2}\right)^{3}+\left(\frac{1}{2}\right)^{4}\right]=-\frac{Q L^{3}}{48 E I},
$$




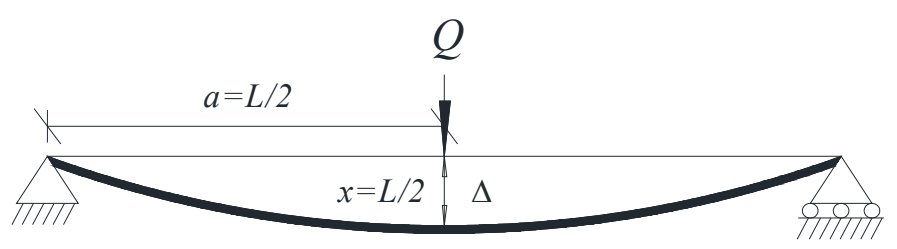

a) Mid-span deflection of beam under a mid-span load

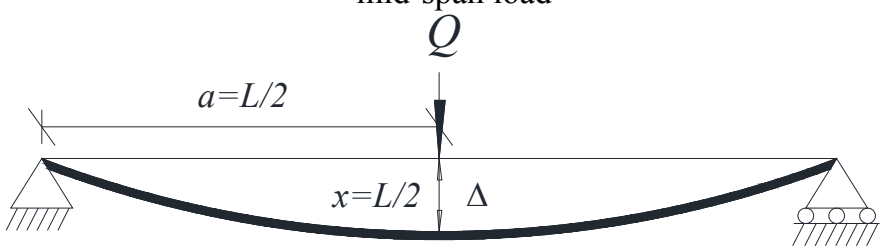

b) Mid-span deflection of beam under a third-point load

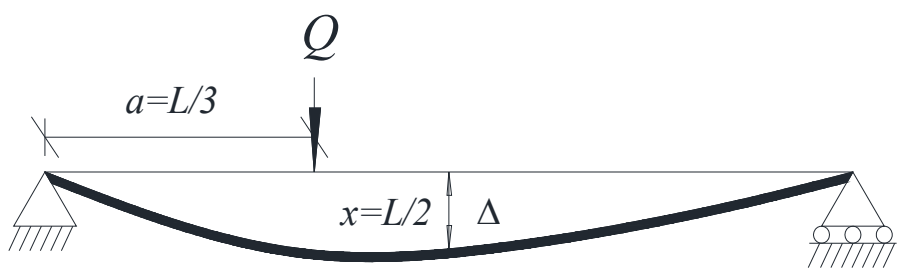

c) Third-point deflection of beam under a third-span load

Figure 5. Simply-supported Beam subjected to a Point Load at Different Locations

which is the same as the theoretical result. For a third-point load (Figure 5(b)), the consistent load and nodal end rotations are obtained from

$$
\frac{E I}{L}\left[\begin{array}{ll}
4 & 2 \\
2 & 4
\end{array}\right]\left\{\begin{array}{l}
\theta_{1} \\
\theta_{2}
\end{array}\right\}=\left\{\begin{array}{c}
-4 Q L / 27 \\
2 Q L / 27
\end{array}\right\} ; \quad\left\{\begin{array}{l}
\theta_{1} \\
\theta_{2}
\end{array}\right\}=\left\{\begin{array}{c}
-5 Q L^{2} / 81 E I \\
4 Q L^{2} / 81 E I
\end{array}\right\}
$$

and so for the consistent load method using a cubic element (Eq. 32)

$$
\left.v\right|_{x=L / 2}=-\frac{5 Q L^{2}}{81 E I}\left(\frac{L}{8}\right)+\frac{4 Q L^{2}}{81 E I}\left(-\frac{L}{8}\right)=-\frac{Q L^{3}}{72 E I}
$$

which differs by $22 \%$ from the exact result $\Delta=-(23 / 1296)\left(Q L^{3} / E I\right)$. Using the present element load method with a higher-order element, it is not necessary to derive a new displacement function, but instead using $a=L / 3$ in Eq. 52 in Appendix 1 gives $\bar{M}_{0}=8 Q L^{2} / 3 E I$ and so, from Eq. 5

$$
\left.v\right|_{x=L / 2}=-\frac{Q L^{3}}{72 E I}-\frac{\left(8 Q L^{2} / 3 E I\right) L}{48}\left[\left(\frac{1}{2}\right)^{2}-2\left(\frac{1}{2}\right)^{3}+\left(\frac{1}{2}\right)^{4}\right]=-\frac{22.5 Q L^{3}}{1296 E I}
$$

which is only $2 \%$ less than the exact solution and clearly much closer than the cubic displacement function. For third-point loading (Figure 5 (c)), the third-point deflection using a cubic element (with Eq. 38 for the rotations) is 
$\left.v\right|_{x=L / 3}=-\frac{5 Q L^{2}}{81 E I}\left(\frac{4 L}{27}\right)+\frac{4 Q L^{2}}{81 E I}\left(-\frac{2 L}{27}\right)=-\frac{28 Q L^{3}}{2187 E I}$

which is $22 \%$ different from the exact result $\Delta=-(36 / 2187)\left(Q L^{3} / E I\right)$. For the higher-order element with the element load effect, $\bar{M}_{0}$ is the same but the new location $x=L / 3$ is used for $N_{q}$, giving

$\left.v\right|_{x=L / 3}=-\frac{28 Q L^{3}}{2187 E I}-\frac{\left(8 Q L^{2} / 3 E I\right) L}{48}\left[\left(\frac{1}{3}\right)^{2}-2\left(\frac{1}{3}\right)^{3}+\left(\frac{1}{3}\right)^{4}\right]=-\frac{34 Q L^{3}}{2187 E I}$,

which is $5.6 \%$ different from the exact result.

\subsubsection{Simply supported beam with trapezoidal loading}

A simply supported beam with distributed loading in two trapezoidal patterns is shown in Figure 6; this example being chosen to demonstrate the use of superposition. For the case in Figure 6(a) where the trapezoidal loading is rectangular, the consistent load and nodal rotations are

$\frac{E I}{L}\left[\begin{array}{ll}4 & 2 \\ 2 & 4\end{array}\right]\left\{\begin{array}{l}\theta_{1} \\ \theta_{2}\end{array}\right\}=\left\{\begin{array}{c}-13 q L^{2} / 324 \\ 13 q L^{2} / 324\end{array}\right\} ; \quad\left\{\begin{array}{l}\theta_{1} \\ \theta_{2}\end{array}\right\}=\left\{\begin{array}{c}-13 q L^{3} / 648 E I \\ 13 q L^{3} / 648 E I\end{array}\right\}$

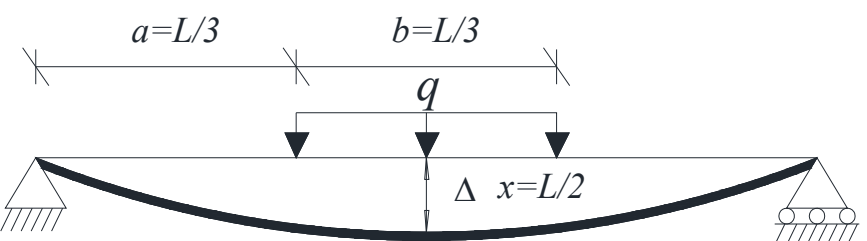

a) Mid-span deflection of beam under partial uniform load

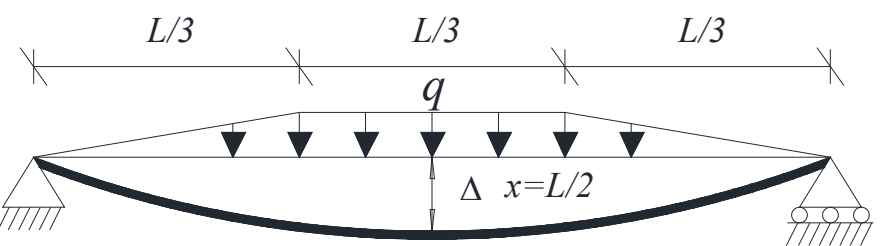

b) Mid-span deflection of beam under trapezoidal load

Figure 6. Simply-supported Beam subjected to various Trapezoidal Loads

and so the mid-span deflection using a cubic element is

$\left.v\right|_{x=L / 2}=-\frac{13 q L^{3}}{648 E I}\left(\frac{L}{8}\right)+\frac{13 q L^{3}}{648 E I}\left(-\frac{L}{8}\right)=-\frac{13 q L^{4}}{2592 E I}$,

which is $24 \%$ different from the exact result $\Delta=-(205 / 31,104)\left(q L^{4} / E I\right)$. The equivalent mid-span moment of Eq. 58 in Appendix 1 when $a=L / 3$ and $b=2 L / 3$ is $\bar{M}_{0}=10 q L^{3} / 9 E I$, producing 


$$
\left.v\right|_{x=L / 2}=-\frac{13 q L^{4}}{2592 E I}-\frac{\left(10 q L^{3} / 9 E I\right) L}{48}\left[\left(\frac{1}{2}\right)^{2}-2\left(\frac{1}{2}\right)^{3}+\left(\frac{1}{2}\right)^{4}\right]=-\frac{201 q L^{4}}{31,104 E I}
$$

for the higher-order element, which is within $1.95 \%$ of the exact result. A typical floor loading pattern is obtained by adding two triangular distributed loading portions to the uniform distribution in Figure 6(a), to produce the pattern in Figure 6(b). For this, the consistent load and associated end rotations are

$$
\frac{E I}{L}\left[\begin{array}{ll}
4 & 2 \\
2 & 4
\end{array}\right]\left\{\begin{array}{l}
\theta_{1} \\
\theta_{2}
\end{array}\right\}=\left\{\begin{array}{c}
-11 q L^{2} / 162 \\
11 q L^{2} / 162
\end{array}\right\} ; \quad\left\{\begin{array}{l}
\theta_{1} \\
\theta_{2}
\end{array}\right\}=\left\{\begin{array}{c}
-11 q L^{3} / 324 E I \\
11 q L^{3} / 324 E I
\end{array}\right\}
$$

for which the mid-span deflection using a cubic element is

$$
\left.v\right|_{x=L / 2}=-\frac{11 q L^{3}}{324 E I}\left(\frac{L}{8}\right)+\frac{11 q L^{3}}{324 E I}\left(-\frac{L}{8}\right)=-\frac{11 q L^{4}}{1296 E I}
$$

which is $22 \%$ different from the exact result $\Delta=-(1681 / 155,520)\left(q L^{4} / E I\right)$. On the other hand, the value of $\bar{M}_{0}$ for the higher-order element can be obtained by adding Eqs. 58, 63 and 68 in Appendix 1, giving

$$
\bar{M}_{0}=\frac{8 q L^{3}}{27 E I}+\frac{10 q L^{3}}{9 E I}+\frac{8 q L^{3}}{27 E I}=\frac{46 q L^{3}}{27 E I}
$$

And Eq. 5 produces

$$
\left.v\right|_{x=L / 2}=-\frac{11 q L^{4}}{1296 E I}-\frac{\left(46 q L^{3} / 27 E I\right) L}{(48+q)}\left[\left(\frac{1}{2}\right)^{2}-2\left(\frac{1}{2}\right)^{3}+\left(\frac{1}{2}\right)^{4}\right]=-\frac{1665 q L^{4}}{155,520 E I},
$$

which is within $0.95 \%$ of the exact result.

In conclusion, the present higher-order element can improve the accuracy of first-order element solution in terms of deflection subjected to the diverse kind of loading patterns remarkably compared to the cubic element. Further, the solutions at other locations seem to be as somewhat less accurate as the solution at mid-span, but these solutions are still regarded as a good agreement with the exact solutions.

\subsection{Numerical Results for Beam-column Deflections with Varied Locations}

The previous study indicated the accuracy and versatility for an elastic beam under a variety of element loading regimes, whose behaviour is first-order. The present example illustrates the deflections with varied locations of a beam-column element under different element loads with and without second-order effects considered. The profound implication of this example is to extend the capability of element solution to the higher degree of accuracy in the field of displacement with recourse to the present analysis with element load effect. On the contrary, one conventional cubic element by virtue of the consistent load method is deficient at evaluating the element solution. Actually, the consistent load method is incapable of predicting the second-order element solution when regardless of equilibrium condition in the assumed finite element function. To this end, this 
example is targeted for demonstration of the present element load method that is valid for the second-order element solution using only one element.

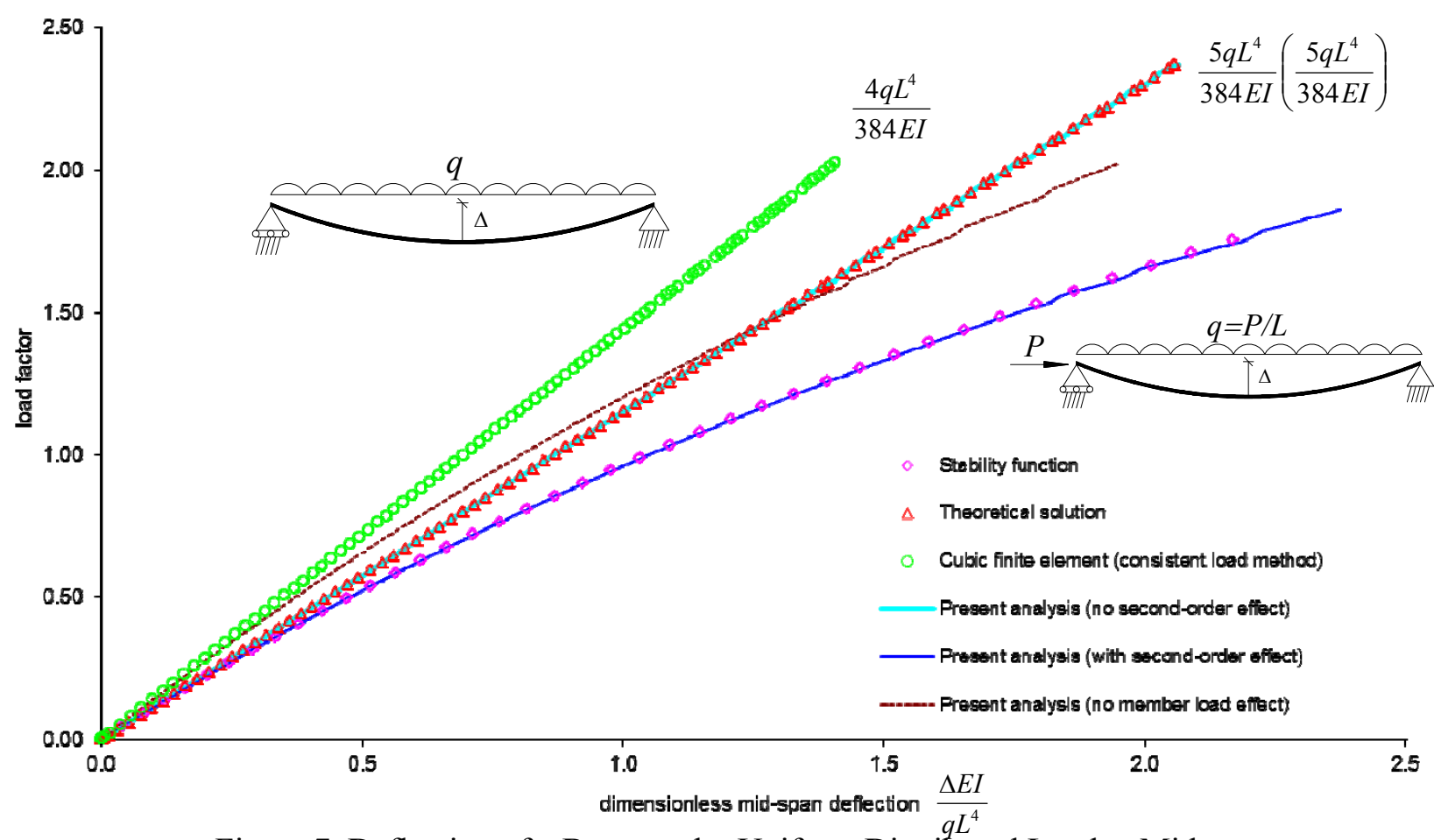

Figure 7. Deflection of a Beam under Uniform Distributed Load at Mid-span

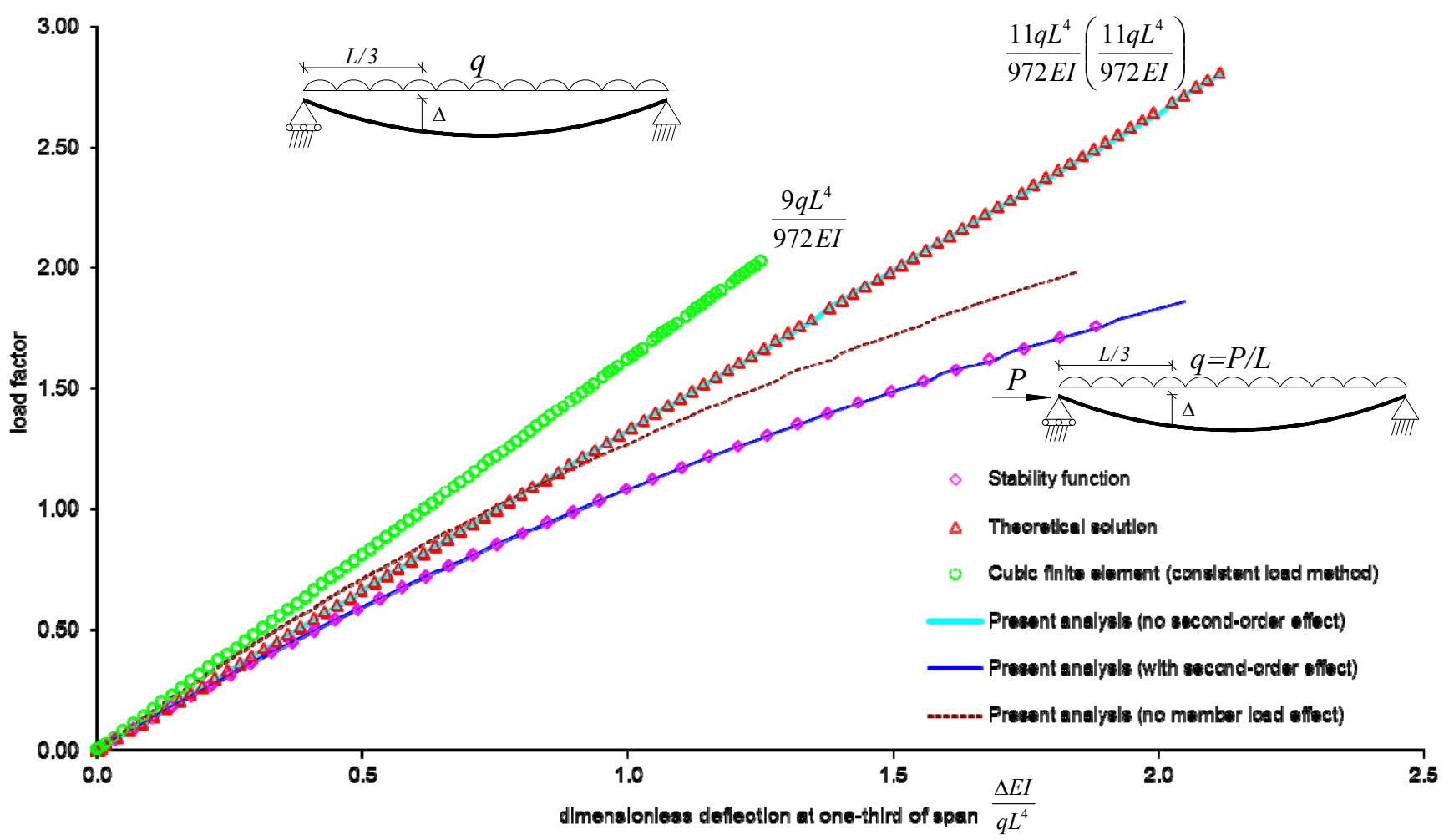

Figure 8. Deflection of a Beam under Uniform Distributed Load at One-third of Span 
Figures 7 and 8 show a simply supported beam subjected to a uniformly distributed load $q(5 \mathrm{kN} / \mathrm{m})$, and they respectively plot the normalised beam deflection at mid-span and one-third of span $E I \Delta / q L^{4}$ against the load factor $\lambda$ whose incremental value complies with various load method. The proposed method is able to produce numerically the accurate deflections at mid-span and one-third of span as depicted in Figures 7 and 8, respectively, whose values are plotted in the figures inside the parenthesis correspondingly, in which the values from cubic element and exact solution from simple beam theory (first-order) are also indicated. On the other hand, one cubic element using consistent load is unable to replicate the first-order theoretical solution as shown in Figures 7 and 8 . In regard to second-order element solution, the present element load method is able to predict the same deflection solution as obtained using the stability functions (second-order), in which coupling between the transverse element load and axial compression is incorporated.

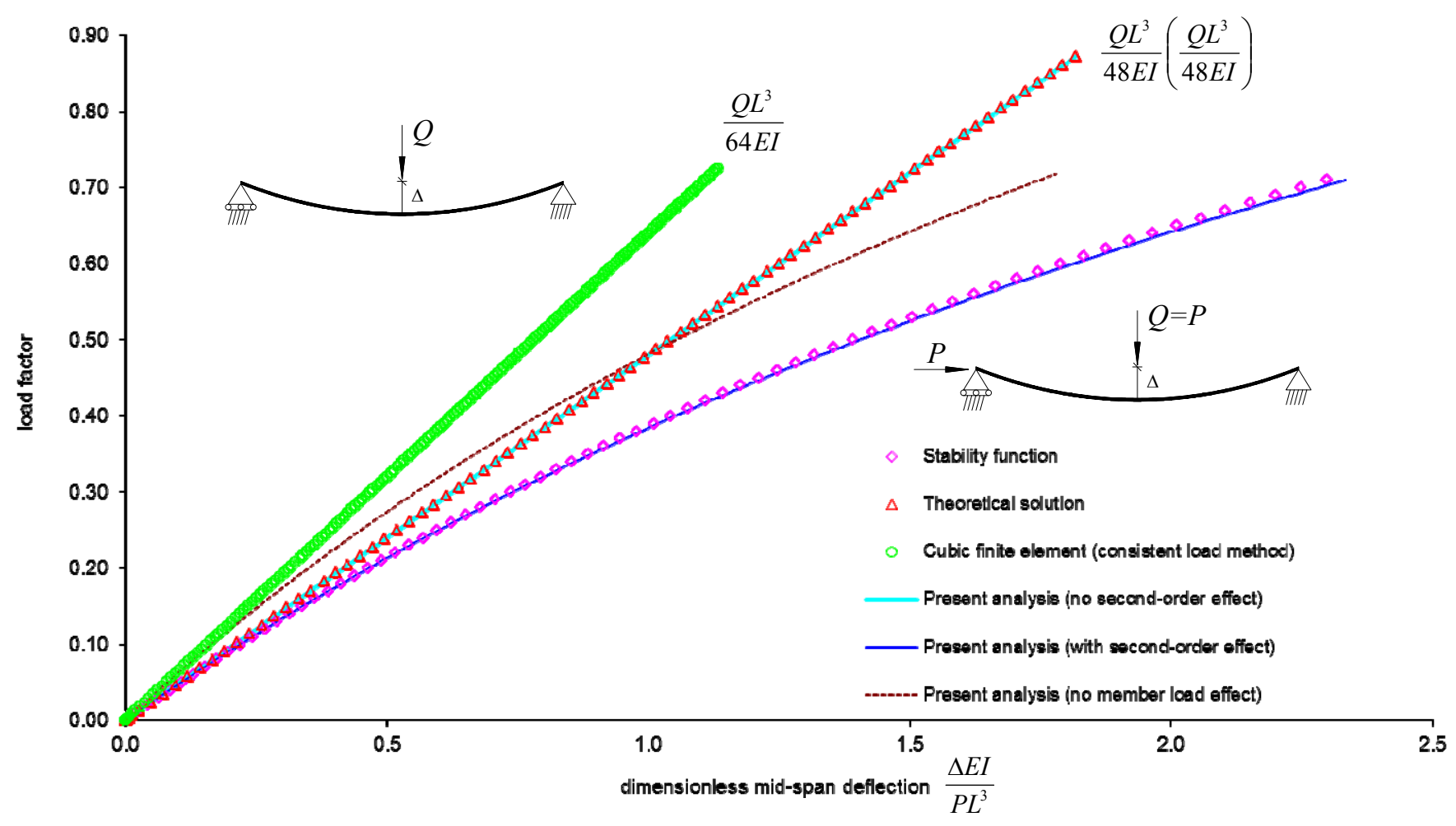

Figure 9. Deflection of a Beam under a Single Point Load at Mid-span

A counterpart analysis with a concentrated load Q $(10 \mathrm{kN})$ at mid-span and one-third of span is presented in Figures 9 and 10, respectively, with the normalised beam deflection $E I \Delta / Q L^{3}$ plotted against the load factor $\lambda$. Similarly, the disparity between cubic element with the consistent load method and theoretical solution are notably present, as indicated in Figures 9 and 10. Their disparity of first-order mid-span deflection is exactly $25 \%$ as also stated in the Section 6.1.2. On the contrary, the first-order deflections at mid-span and one-third of span from the present analysis, which display inside parenthesis in Figures 9 and 10, respectively, are both close to the exact solutions. 


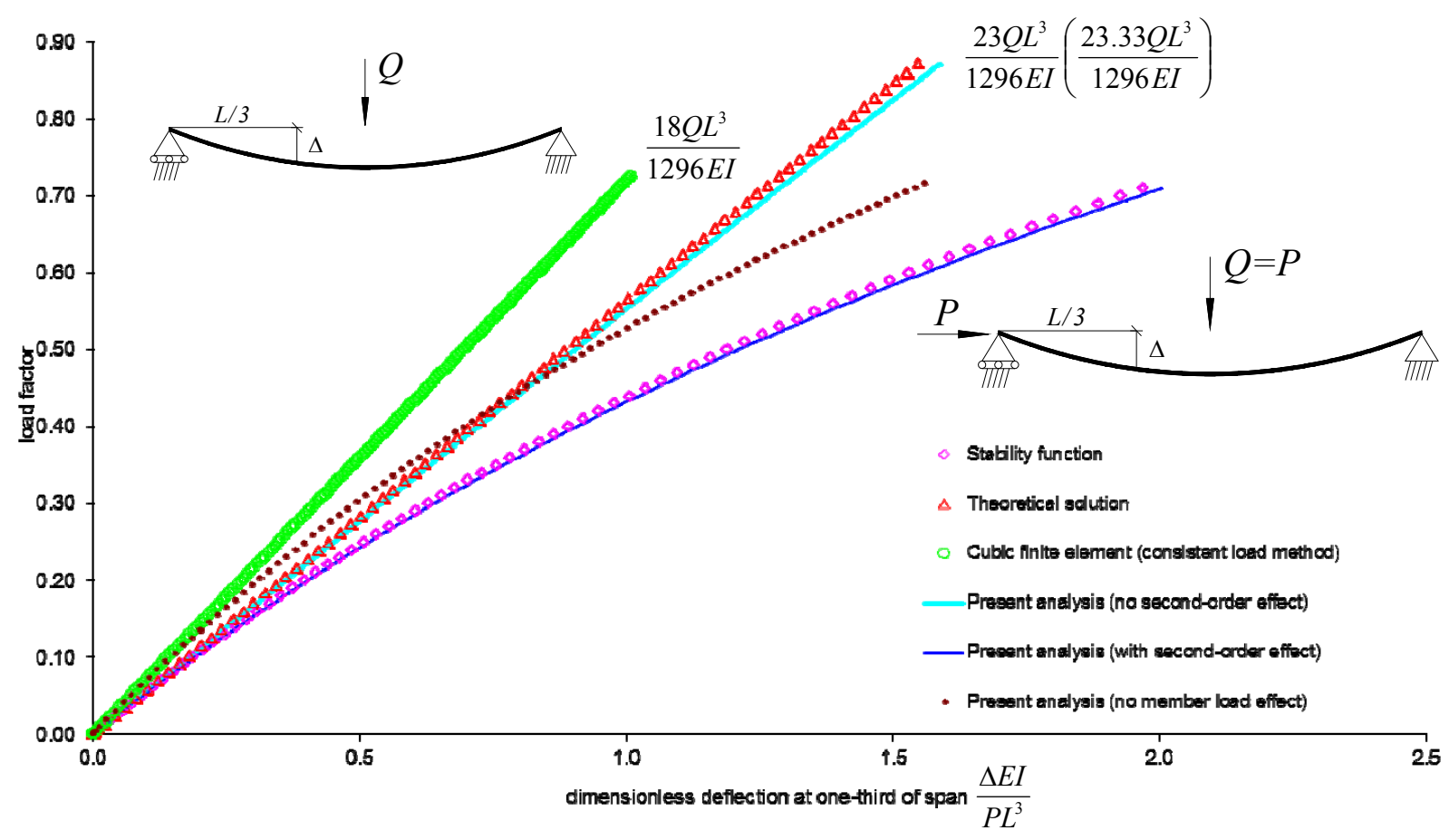

Figure 10. Deflection of a Beam under a Single Point Load at One-third of Span

A beam with two point loads Q $(10 \mathrm{kN})$ located at quarter points is shown in Figures 11 and 12 for the load-deflection solution at mid-span and a quarter of span in order to demonstrate the principle of superposition adopted in the numerical formulation. The normalised deflections $E I \Delta / Q L^{3}$ at mid-span and a quarter of span against load factor $\lambda$ from present analysis is respectively plotted in the Figures 11 and 12 are shown to be in good agreement with the exact solution for first-order analysis except the cubic element using consistent load method. Their first-order deflections from the present analysis are shown inside the parenthesis in the corresponding figures. The axial force introduces second-order behaviour into the element solution, and its solution is the same as that determined from the stability functions. Therefore, the present approach with the element load effect can successfully demonstrate its accuracy of the first-order element load effect as well as second-order coupling effect. 


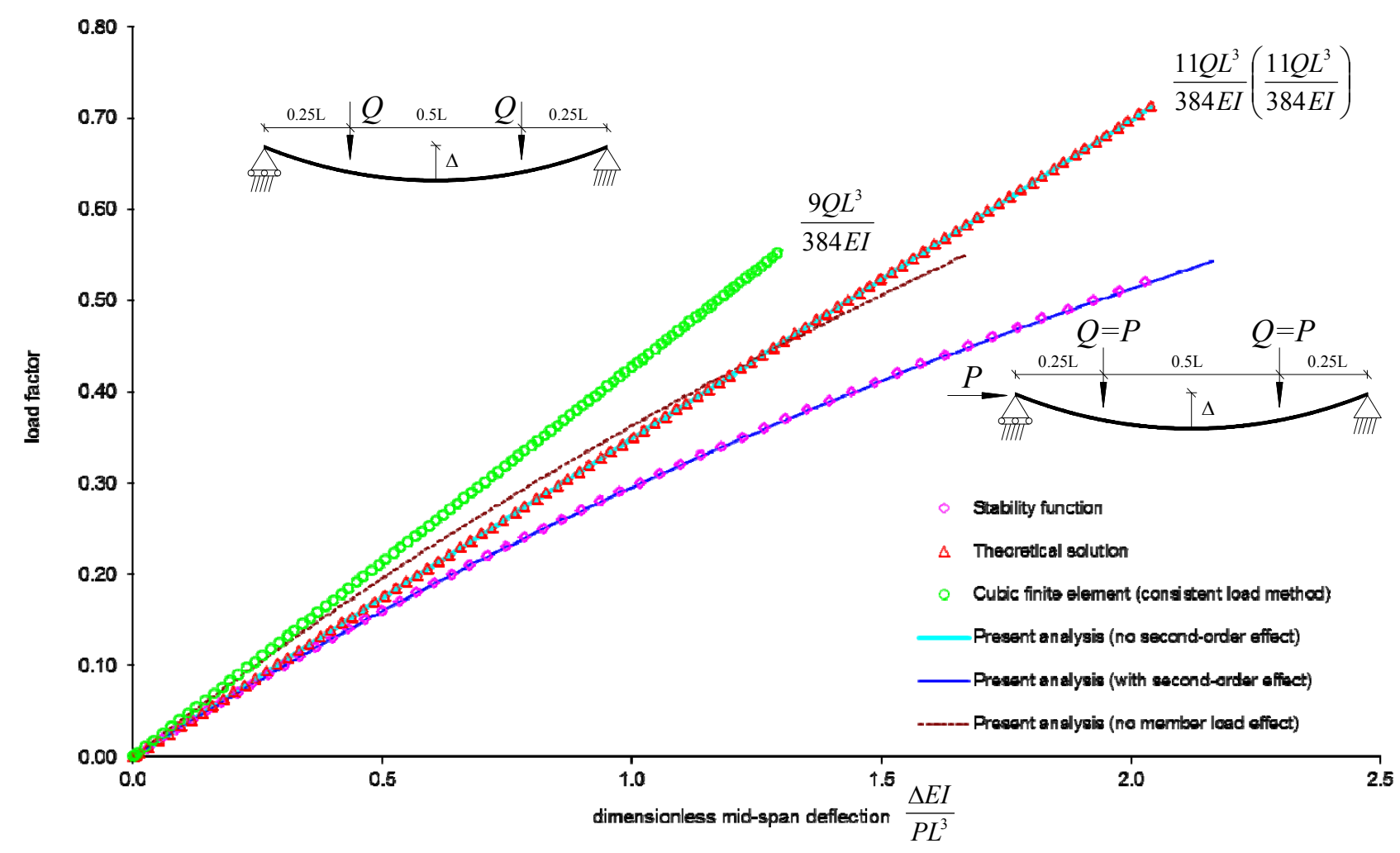

Figure11. Deflection of a Beam under Two Point Loads at Mid-span

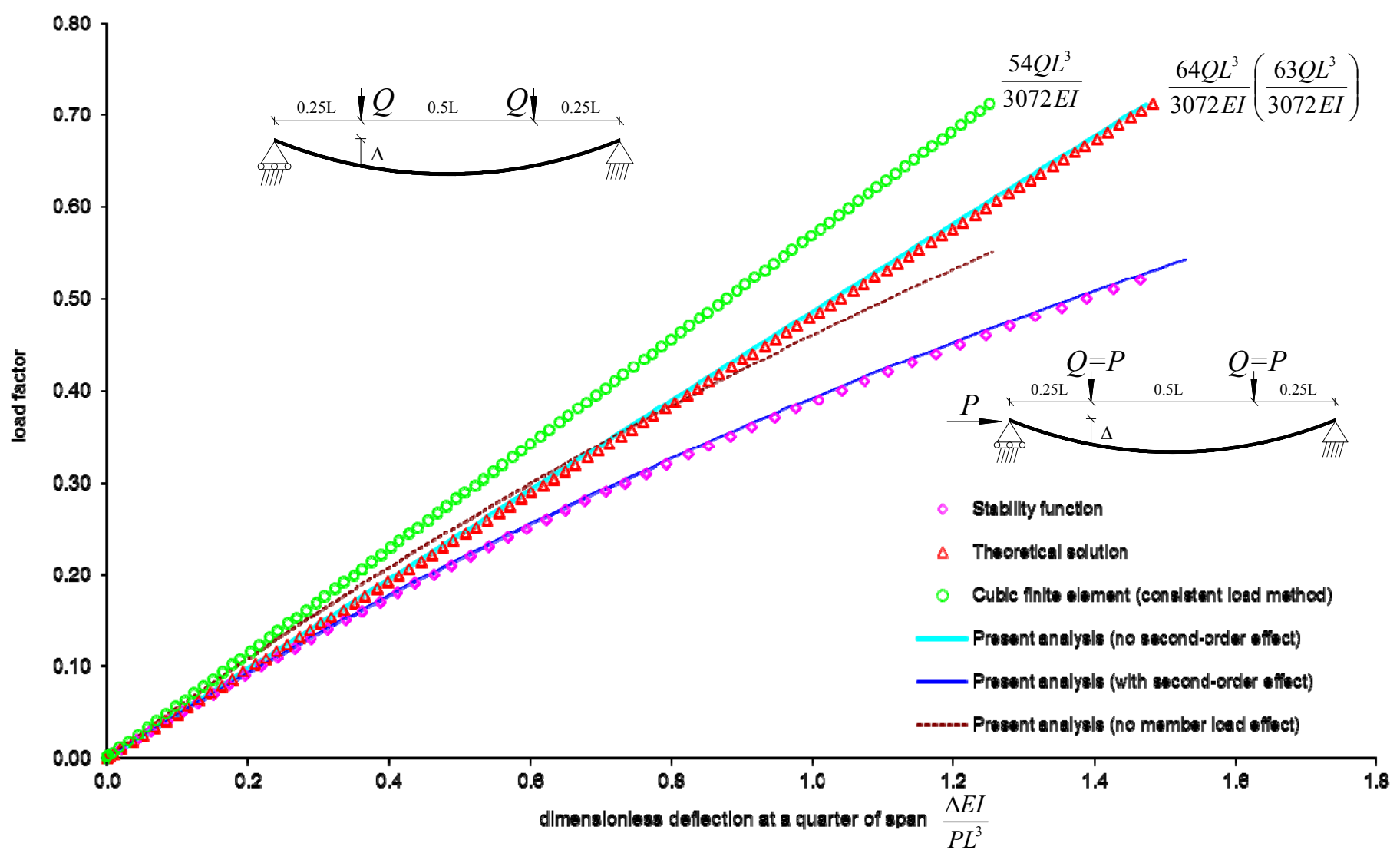

Figure12. Deflection of a Beam under Two Point Loads at a Quarter of Span 
It should be remarked that, according to the present analysis with element load effect, the discrepancy between the deflections at mid-span and other locations are very insignificant as similarly demonstrated in the previous example that the first-order deflections were studied. It can be concluded that, despite the sacrifice of load distribution effect at other locations but mid-span, the present analysis with element load effect can still produce accurate element solution in terms of displacements along an entire element. Therefore, the present analysis with element load effect is very capable of analysing the whole element solution of either a bending beam or a typical buckling member.

Further, this example can demonstrate that a single present higher-order element function with element load effect can generalize and replace the plethora of stability functions with different element loads, in which the present element load method is same but changing the magnitude of the equivalent mid-span moment coefficient $\bar{M}_{0}$ only in a robust manner. In summary, the stability functions with a plethora of element load scenarios can be transformed into a single present generalized element load method without loss of accuracy along an element itself.

\subsection{Postbuckling of Right-angled Frame}

Roorda [13] and Koiter [14] provided the first experimental and analytical results respectively for the right-angled frame shown in Figure 13, with the analytical formulation accounting for member bowing and for postbuckling. This structure was later studied by Argyris and Dunne [15] and Chan and Zhou [2]. The right-angled frame in Figure 13 with pin supports was analysed herein with a member point load $P$ at an eccentricity of $e=254 \mathrm{~mm}$ (10 inch), applied directly to the beam without resolving it as an eccentric moment and point loads at the element nodes. The cross-section, geometry and material properties of the frame are given in Figure 13, which also plots the joint rotation $\theta$ against the dimensionless load $P / P_{E}$, where $P_{E}$ is the Euler load. The proposed non-linear modelling using only one element produces results which are in close coincidence with those of Chan and Zhou [2], as well as approaching the postbuckling response of the perfect frame $(e=0)$ given by Koiter. This example validates that the present approach is capable of accounting for the element load effect including member bowing and postbuckling of a simple framed structure in which load transformation is needed between member and global coordinates. It should be noted that the present analysis produces slightly larger joint rotations than those obtained by Iu and Bradford [6] which utilise lumped loading, as the axial load due to element load effect produced in the beam due to its restraint induces a further minor second-order element load effect. 


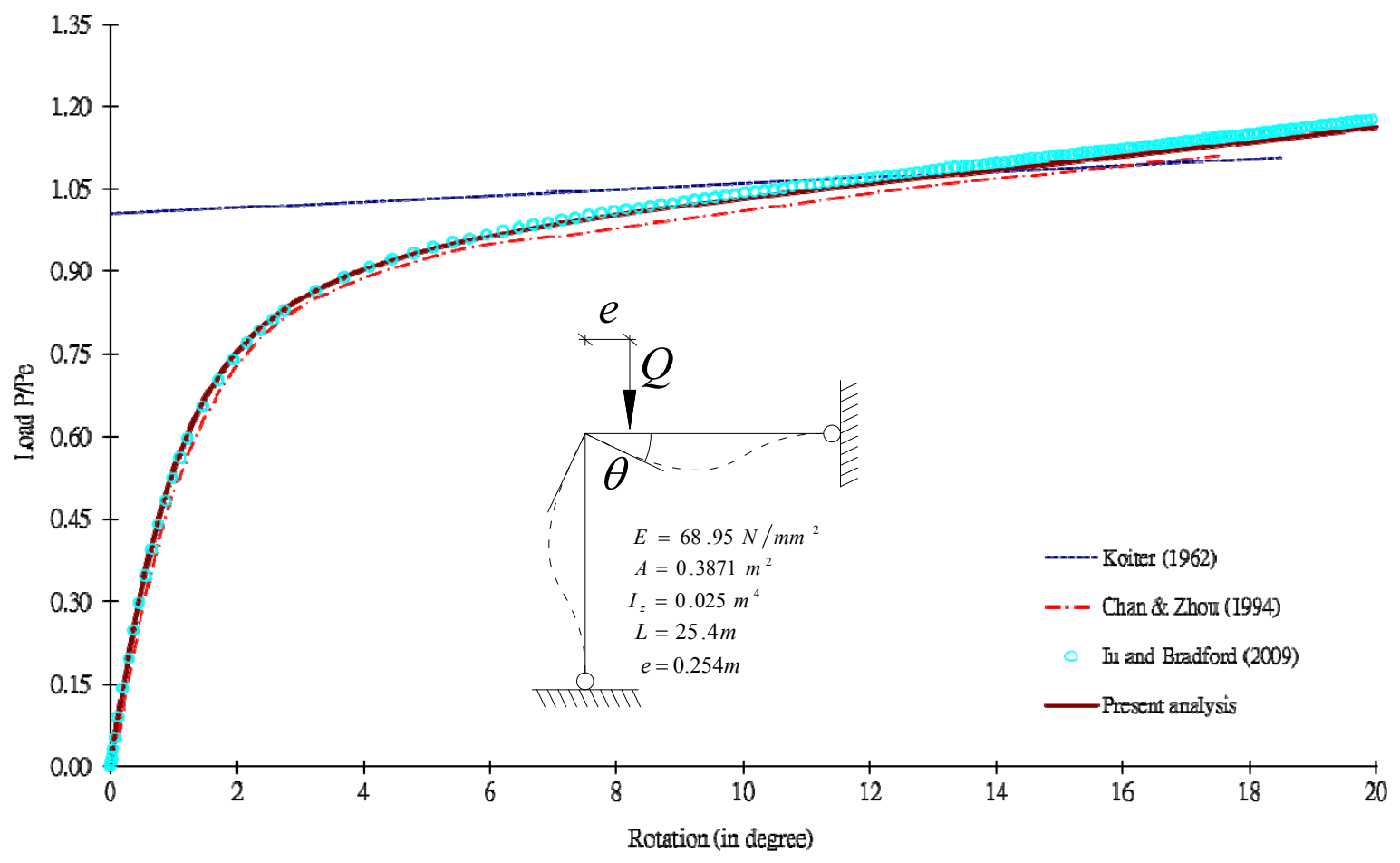

Figure 13. Load-deformation Response of Right-angle Frame

\subsection{Two-storey Rigid Sway Frame}

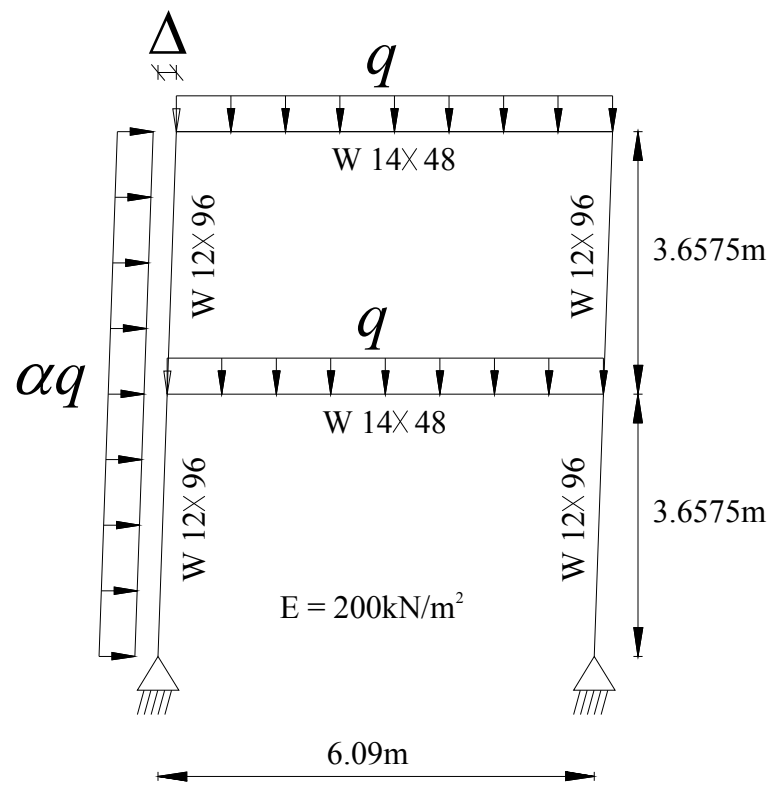

Figure 14. Geometry of Two-storey Building Frame 
Applied forces in practical engineering frames such as wind load, imposed live load and dead load commonly act along the elements, and these loads contributed to sway effect in rigid frames [16]. The present element load method is important, therefore, to be able to use an element permitting element load effects in second-order frame analysis. The two-storey frame shown in Figure 14 has been studied; this frame was also analysed by Zhou and Chan [9] and its geometry, cross-sections and material properties are given in Figure 15. The frame is subjected to uniformly distributed gravity loading $q$ on both beams and to a lateral (wind) loading $\alpha q$, where $\alpha$ is taken as $10^{-3}, 10^{-2}$ and $10^{-1}$.

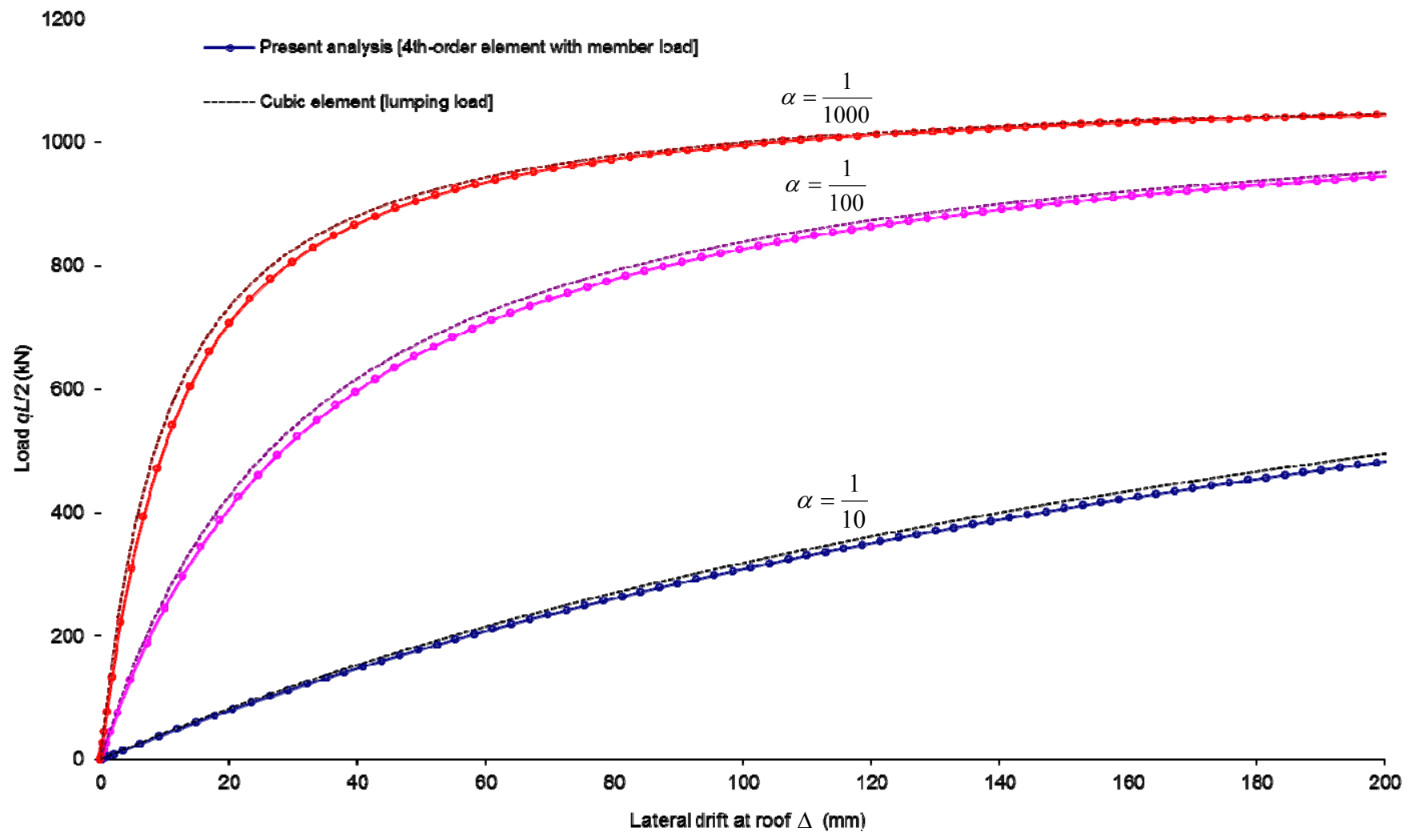

Figure 15. Lateral Drift $\Delta$ and Load Factor Relationship for Two Storey Frame

Figure 15 shows the sway behaviour in terms of the lateral drift of the roof of the building, which is affected by both $\mathrm{P}-\delta$ and P- $\Delta$ responses. The lateral force parameter $\alpha$ has a large influence on the frame behaviour, with the structural responses being different for the three values of $\alpha$ considered. It can be seen, however, that the discrepancies between the results using the lumped loads and the present element load approach for each value of $\alpha$ are not overly large. This is because the most significant contribution to the P- $\Delta$ sway effect is the lateral force rather than nodal moments that are neglected in the lumping load method, so that the lumping load method retains the important lateral force effects and its effect especially for low axial forces is very minor. With larger loads the discrepancy increases owing to the coupling effect between the element load effect and the element stiffness, as in Section 6.2. 


\section{DISCUSSION AND CONCLUDING REMARKS}

This paper presents a profound impact on shifting the nodal solution (robust system analysis) to both nodal and element solution (sophisticated element formulation) and opens a door to study the element solution using an element itself, when element load directly acting on an element is ubiquitous. For the traditional numerical approach, a whole domain must be divided into sub-domain, and the approximate function aims at reproduce the accurate solution for this sub-domain, unfortunately, restrictive to the nodal solution through the system analysis. Therefore, the present analysis provides an alternative but unique means to study first- and second-order element solution effectively without element discretization.

This paper possesses another important implication of superimposition principle being imposed in the derivation of element stiffness formulation that the numerous number of general element loading scenarios can be simply and succinctly unified from a few of standard individual load cases afore non-linear analysis, during which the element loading distribution of any kinds is converted into the standard loading magnitude at mid-span. Meanwhile, this magnitude of element load coefficient, such as $\bar{M}_{0}$, updates in the course of the non-linear solution procedures for the sake of tracing second-order element solution. As a result, despite trading off the element load distribution for that the element stiffness with element load effect is unnecessarily reformulated for a diverse kind of element load cases, the present numerical analysis is therefore versatile and adaptive to a structure under diverse element loading types and scenarios without loss of accuracy considerably.

In addition, in contrast to the numerous stability functions with a plethora element load scenarios, the present higher-order element functions is basically same but adjusting the equivalent mid-span moment $\bar{M}_{0}$ for the corresponding element load cases, but results in an accurate solution as the stability functions as elaborated in Section 6.2. In contrast to the conventional finite element being irrelevant to element loads, such as the cubic element or other advanced finite elements in the open literature, in which all element load effect taking into account at nodes through the system analysis without considering the element solutions, the present higher-order element can accurately evaluate the first- and second-order elastic element solution by element itself when subjected to element loads. In short, the present higher-order element function with element load effect can generalize and replace the numerous stability functions with a plethora of element load scenarios and its load combinations in a simple, efficient, effective, versatile and robust manner.

In conclusion, based on all above mentioned benign features and advances, the present second-order elastic analysis with element; load solution is adequately articulated as being an accurate (solution), simple (formulation), versatile (applications and non-linear behaviour), efficacious (computational speed) and effective (numerical modelling and computational storage) approach, which is favourable to the practical applications for the general steel structures subjected to the multiplicity of random loading cases; especially the reliable structural safety and adequacy of an element (member) can be assured.

\section{ACKNOWLEDGMENT}

The work in this paper was supported by the Australian Research Council through a Federation Fellowship awarded to the second author. Further, the gratitude is given to the School of Civil Engineering and Built Environment of QUT in support to the first author. 


\section{REFERENCES}

[1] Kondoh, K., Tanaka, K. and Atluri, S.N., "An Explicit Expression for the Tangent-stiffness of a Finitely Deformed 3-D Beam and its use in the Analysis of Space Frames", Computers and Structures 2006, Vol. 24, No. 2, pp. 253-271.

[2] Chan, S.L. and Zhou, Z.H., "Pointwise Equilibrating Polynomial Element for Nonlinear Analysis of Frames", Journal of Structural Engineering, ASCE 1994, Vol. 120, No. 6, pp. 1703-1717.

[3] Chan, S.L. and Zhou, Z.H., "Second-order Elastic Analysis of Frames using Single Imperfect Element per Member", Journal of Structural Engineering, ASCE 1995, Vol. 121, No. 6, pp. 939-945.

[4] Izzuddin, B.A., "Quartic Formulation for Elastic Beam-columns subject to Thermal Effects", Journal of Engineering Mechanics, ASCE 1996, Vol. 122, No. 9, pp. 861-871.

[5] Liew, J.Y.R., Chen, H., Shanmugam, N.E. and Chen, W.F., "Improved Nonlinear Plastic Analysis of Space Frame Structures", Engineering Structures 2000, Vol. 22, pp. 1324-1338.

[6] Iu, C.K. and Bradford, M.A., "Second-order Elastic Analysis of Steel Structures using a Single Element per Member", Engineering Structures 2010, Vol. 32, pp. 2606-2616.

[7] Iu, C.K. and Bradford, M.A., "Higher-order Non-linear Analysis of Steel Structures Part I: Elastic Second-order Formulation”, International Journal of Advanced Steel Construction 2012, Vol. 8, No. 2, pp. 168-182.

[8] Iu, C.K. and Bradford, M.A., "Higher-order Non-linear Analysis of Steel Structures Part II: Refined Plastic Hinge Formulation", International Journal of Advanced Steel Construction 2012, Vol. 8, No. 2, pp. 183-198.

[9] Zhou, Z.H. and Chan, S.L., "Refined Second-order Analysis of Frames with Members under Lateral and Axial Loads", Journal of Structural Engineering, ASCE 1996, Vol. 122, No. 5, pp. 548-554.

[10] Zhou, Z.H. and Chan, S.L., "Second-order Analysis of Slender Steel Frames under Distributed Axial and Member Loads", Journal of Structural Engineering, ASCE 1997, Vol. 123, No. 9, pp. 1187-1193.

[11] Kassimali, A., "Large Deformation Analysis of Elastic-plastic Frames", Journal of Structural Engineering, ASCE 1983, Vol. 109, No. 8, pp. 1869-1886.

[12] Meek, J.L. and Tan, H.S., "Geometrically Nonlinear Analysis of Space Frames by an Incremental Iterative Technique", Computer Methods in Applied Mechanics and Engineering 1984, Vol. 47, pp. 261-282.

[13] Roorda, J., "Stability of Structures with Small Imperfections", Journal of the Engineering Mechanics Division, ASCE 1965, Vol. 91, No. 1, pp. 87-106.

[14] Koiter, W.T., "Post-buckling Analysis of a Simple Two-bar Frame", Recent Progress in Applied Mechanics (Broberg et al. eds), John Wiley \& Sons, New York, 1967, pp. 337-354.

[15] Argyris, J.H. and Dunne, P.C., "On the Application of the Natural Mode Technique to Small Strain Large Displacement Problems", Proceedings of World Congress on Finite Element Methods in Structural Mechanics, Bournemouth, UK, 1975.

[16] Trahair, N.S., Bradford, M.A., Nethercot, D.A. and Gardner, L., "The Behaviour and Design of Steel Structures to EC3”, $4^{\text {th }}$ edn., Taylor \& Francis, London, 2008. 
Appendix 1. Equivalent Mid-Span Moment $\bar{M}_{0}$

\section{Concentrated moment}

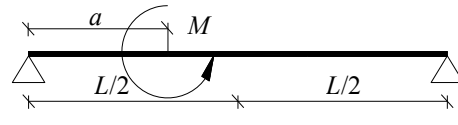

$\bar{M}_{0}=-\frac{L}{E I} \times 8 M$

$\bar{M}_{0}=\frac{L}{E I} \times 8 M$

\section{Point load}

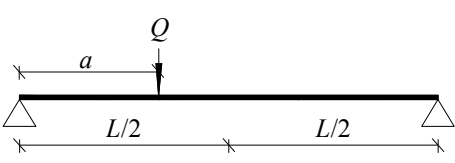

$\bar{M}_{0}=\frac{L}{E I} \times 8 Q a$

$\bar{M}_{0}=\frac{L}{E I} \times 8 Q(L-a)$

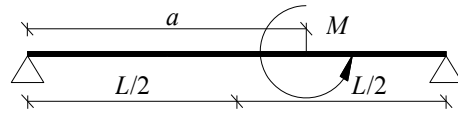

$(a \leq L / 2)$

$(a \geq L / 2)$

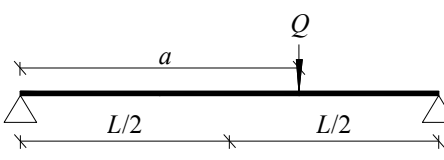

$(a \leq L / 2)$

$(a \geq L / 2)$

3. General $\boldsymbol{n}$ point loads

$$
\begin{array}{ll}
\bar{M}_{0}=\frac{L}{E I} \sum_{i=1}^{j} 8 Q_{i} a_{i} & \left(a_{j} \leq L / 2\right) \\
\bar{M}_{0}=\frac{L}{E I} \sum_{i=j+1}^{n} 8 Q_{i}\left(L-a_{i}\right) & \left(a_{j} \geq L / 2\right)
\end{array}
$$

\section{Uniformly distributed load over entire length}

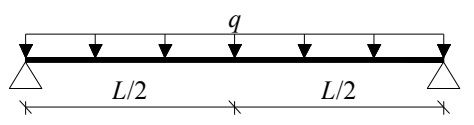

$\bar{M}_{0}=\frac{L}{E I} \times 2 q L^{2}$

\section{Uniformly distributed load over partial length}
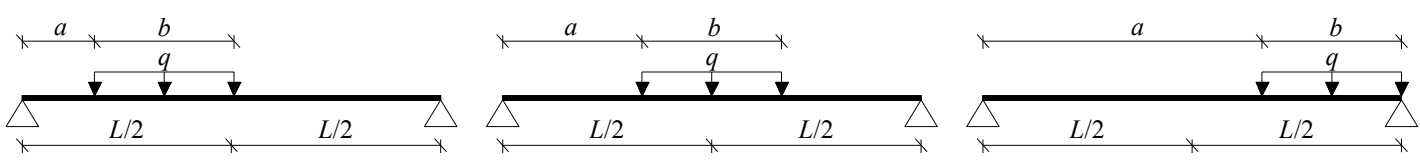

$\bar{M}_{0}=\frac{L}{E I} \times 8 q b\left(a+\frac{b}{2}\right)$

$(a+b \leq L / 2)$

$\bar{M}_{0}=\frac{L}{E I} \times 8 q\left[b\left(L-\left(a+\frac{b}{2}\right)\right)-\left(\frac{L}{2}-a\right)^{2}\right] \quad(a \leq L / 2 \leq a+b)$ 


$$
\bar{M}_{0}=\frac{L}{E I} \times 8 q b\left(L-\left(a+\frac{b}{2}\right)\right) \quad(a \geq L / 2)
$$

6. General $n$ uniform loads

$$
\begin{array}{ll}
\bar{M}_{0}=\frac{L}{E I} \times 8 \sum_{i=1}^{j-1} q_{i} b_{i}\left(a_{i}+\frac{b_{i}}{2}\right) & (a+b \leq L / 2) \\
\bar{M}_{0}=\frac{L}{E I} \times 8 q_{j}\left[b_{j}\left(L-\left(a_{j}+\frac{b_{j}}{2}\right)\right)-\left(\frac{L}{2}-a_{j}\right)^{2}\right] & (a \leq L / 2 \leq a+b) \\
\bar{M}_{0}=\frac{L}{E I} \times 8 \sum_{i=j+1}^{n} q_{i} b_{i}\left(L-\left(a_{i}+\frac{b_{i}}{2}\right)\right) & (a \geq L / 2)
\end{array}
$$

\section{Hydrostatic loading}
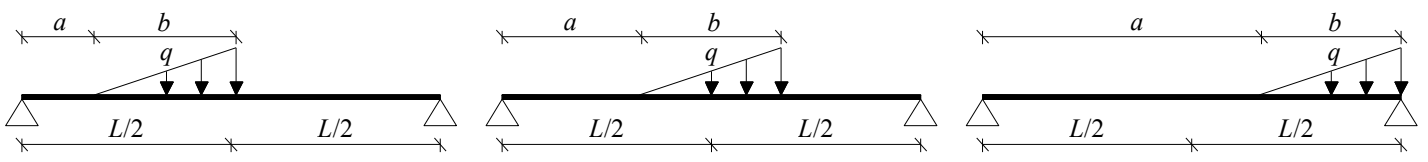

$$
\begin{array}{ll}
\bar{M}_{0}=\frac{L}{E I} \times 4 q b\left(a+\frac{2 b}{3}\right) & (a+b \leq L / 2) \\
\bar{M}_{0}=\frac{L}{E I} \times 8 q\left[\frac{b}{2}\left(L-\left(a+\frac{2 b}{3}\right)\right)-\frac{(L / 2-a)^{3}}{3 b}\right] & (a \leq L / 2 \leq a+b) \\
\bar{M}_{0}=\frac{L}{E I} \times 4 q b\left(L-\left(a+\frac{2 b}{3}\right)\right) & (a \geq L / 2)
\end{array}
$$
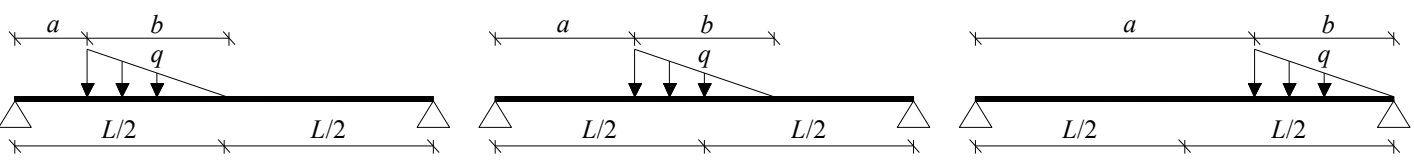

$$
\begin{array}{ll}
\bar{M}_{0}=\frac{L}{E I} \times 4 q b\left(a+\frac{b}{3}\right) & (a+b \leq L / 2) \\
\bar{M}_{0}=\frac{L}{E I} \times 8 q\left[\frac{b}{2}\left(a+\frac{b}{3}\right)-\frac{(L / 2-a)^{2}(L / 2-a+b)}{3 b}\right] & (a \leq L / 2 \leq a+b) \\
\bar{M}_{0}=\frac{L}{E I} \times 4 q b\left(L-\left(a+\frac{b}{3}\right)\right) & (a \geq L / 2)
\end{array}
$$




\section{Appendix 2. Stiffness Terms}

The terms $G_{\alpha i}(\alpha=y$ or $z, i=1$ or 2$)$ in Eq. 26 are:

$$
\begin{aligned}
& \frac{\partial M_{\alpha 1}}{\partial \omega}=\frac{E I_{\alpha}}{L}\left\{\left[\frac{12(48)^{2} / 5+36(48) \omega / 5+252 \omega^{2} / 35+\omega^{3} / 20}{(48+\omega)^{3}}\right]\left(\theta_{\alpha 1}+\theta_{\alpha 2}\right)\right. \\
& +\left[\frac{4(48)^{2}+28(48) \omega / 5+132 \omega^{2} / 35+11 \omega^{3} / 420}{(48+\omega)^{3}}\right]\left(\theta_{\alpha 1}-\theta_{\alpha 2}\right) \\
& \left.+\left[\frac{16 \omega}{35(48+\omega)^{3}} \bar{M}_{0}\right]\right\} \\
& =\frac{E I_{\alpha}}{L}\left\{2 b_{1}\left(\theta_{\alpha 1}+\theta_{\alpha 2}\right)+2 b_{2}\left(\theta_{\alpha 1}-\theta_{\alpha 2}\right)+b_{w 1} \bar{M}_{0}\right\}=\left(\frac{E I_{\alpha}}{L}\right) G_{\alpha 1} \\
& \frac{\partial M_{\alpha 2}}{\partial \omega}=\frac{E I_{\alpha}}{L}\left\{\left[\frac{12(48)^{2} / 5+36(48) \omega / 5+252 \omega^{2} / 35+\omega^{3} / 20}{(48+\omega)^{3}}\right]\left(\theta_{\alpha 1}+\theta_{\alpha 2}\right)\right. \\
& -\left[\frac{4(48)^{2}+28(48) \omega / 5+132 \omega^{2} / 35+11 \omega^{3} / 420}{(48+\omega)^{3}}\right]\left(\theta_{\alpha 1}-\theta_{\alpha 2}\right) \\
& \left.-\left[\frac{16 q}{35(48+\omega)^{3}}\right] \bar{M}_{0}\right\} \\
& =\frac{E I_{\alpha}}{L}\left\{2 b_{1}\left(\theta_{\alpha 1}+\theta_{\alpha 2}\right)-2 b_{2}\left(\theta_{\alpha 1}-\theta_{\alpha 2}\right)-b_{w 1} \bar{M}_{0}\right\}=\left(\frac{E I_{\alpha}}{L}\right) G_{\alpha 2} \\
& \frac{\partial \omega}{\partial e}=\frac{1 / L}{I / A L^{2}-\sum_{\alpha=y, z}\left[b_{2}^{\prime}\left(\theta_{\alpha 1}-\theta_{\alpha 2}\right)^{2}+b_{w 1}^{\prime}\left(\theta_{\alpha 1}-\theta_{\alpha 2}\right) \bar{M}_{0}+b_{w 2}^{\prime} \bar{M}_{0}^{2}\right]}=\frac{1}{H L} \\
& \frac{\partial \omega}{\partial \theta_{\alpha 1}}=\frac{2 b_{1}\left(\theta_{\alpha 1}+\theta_{\alpha 2}\right)+2 b_{2}\left(\theta_{\alpha 1}-\theta_{\alpha 2}\right)+b_{w 1} \bar{M}_{0}}{I / A L^{2}-\sum_{\alpha=y, z}\left[b_{2}^{\prime}\left(\theta_{\alpha 1}-\theta_{\alpha 2}\right)^{2}+b_{w 1}^{\prime}\left(\theta_{\alpha 1}-\theta_{\alpha 2}\right) \bar{M}_{0}+b_{w 2}^{\prime} \bar{M}_{0}^{2}\right]}=\frac{G_{\alpha 1}}{H} \\
& \frac{\partial \omega}{\partial \theta_{\alpha 2}}=\frac{2 b_{1}\left(\theta_{\alpha 1}+\theta_{\alpha 2}\right)-2 b_{2}\left(\theta_{\alpha 1}-\theta_{\alpha 2}\right)-b_{w 1} \bar{M}_{0}}{I / A L^{2}-\sum_{\alpha=y, z}\left[b_{2}^{\prime}\left(\theta_{\alpha 1}-\theta_{\alpha 2}\right)^{2}+b_{w 1}^{\prime}\left(\theta_{\alpha 1}-\theta_{\alpha 2}\right) \bar{M}_{0}+b_{w 2}^{\prime} \bar{M}_{0}^{2}\right]}=\frac{G_{\alpha 2}}{H}
\end{aligned}
$$

in which

$$
\begin{aligned}
& b_{2}^{\prime}=\left[\frac{-16(48)^{2} / 5-64(48) \omega / 35}{(48+\omega)^{4}}\right] \\
& b_{w 1}^{\prime}=\left[\frac{16(48)-32 \omega}{35(48+\omega)^{4}}\right] \\
& b_{w 2}^{\prime}=\left[\frac{4 / 7+2 q / 105}{(48+q)^{4}}\right] .
\end{aligned}
$$

